\title{
Numerical modelling of melting and solidification problems-A review
}

\author{
BISWAJIT BASU ${ }^{1}$ and A W DATE ${ }^{2}$ \\ ${ }^{1}$ Tata Research Development \& Design Centre, 1, Mangaldas Road, \\ Pune 411001 , India \\ ${ }^{2}$ Department of Mechanical Engineering, Indian Institute of Techno- \\ logy, Bombay 400076 , India
}

MS received 27 February 87; revised 30 November 1987

\begin{abstract}
A generalised mathematical formulation is provided for any melting and solidification problem. Non-dimensional parameters governing this problem are identified. Depending on the problem, ways of simplifying the generalised mathematical statement are discussed. Different methods of formulation of melting and solidification problems, namely, variable and fixed domain methods, are discussed highlighting their merits and demerits. Methods to solve the momentum equation in the molten region and model alloy solidification problems are presented. Recent progress in all the numerical methods is reviewed. Critical numerical aspects of all the methods are discussed. Guidelines are provided to select the correct numerical method. Areas needing emphasis in future research are highlighted.
\end{abstract}

Keywords. Melting and solidification; mathematical formulation; governing equations; interface; mushy region; variable domain; fixed domain; numerical scheme.

\section{Introduction}

\subsection{Phenomena of solidification and melting}

Solidification and melting are commonly observed phenomena. Melting of snow, formation of ice cubes in a domestic refrigerator, melting of iron, gold or silver for making domestic utensils, ornaments or machine components by forging, casting or welding are all common knowledge.

The important feature of the phenomenon however is that it is brought about by a process of heat transfer that is accompanied by a change of phase i.e. from solid to liquid or vice versa. During a phase change, thermal energy is released or

A list of symbols is given at the end of the paper 
absorbed at the interface between the solid and the liquid. This energy, which is also known as the latent heat of fusion $(\lambda)$, is drawn from one phase and distributed through the other primarily by a process of conduction heat transfer. Under certain circumstances, however, the liquid phase may not remain stagnant and the essentially conduction heat transfer may be superimposed by convective heat transfer.

It is well known that whereas the transfer of heat by confuction results from temperature differences within each phase, the release or absorption of latent heat at the interface occurs without any temperature difference at all. It is this characteristic of latent heat which makes the phenomenon of solidification and melting a transient one.

In pure substances, the temperature at which the latent heat is released is uniquely defined and is known as the melting point $\left(T_{m}^{*}\right)$ of the substance. Thus, the interface between the solid and the liquid phases can be sharply identified. Both the $T_{m}^{*}$ and $\lambda$ are properties of a substance. In impure substances or alloys, on the other hand, the total latent heat is released or absorbed over a range of temperatures. The exact amounts of latent heat released at each temperature within the range are however rarely known precisely. Thus the interface between solid and liquid in alloys is not a clearly identifiable surface, but essentially a region of finite thickness. This region is often referred to as the "mushy" zone.

In many applications that will be mentioned in the next section, the engineer needs to know the rates of solidification or melting. This means that he needs to calculate:

a) the rate of heat transfer in materials undergoing solidification and melting;

b) the rate of interface movement (or interface velocity) which yields the estimate of total solidification or melting times in materials of finite volume;

c) the shape of the interface at every instant of time.

The general problem of predicting rates of solidification and melting is known as the "Stefan problem", named after Stefan (1891) who carried out a study of the melting of polar ice. He showed that the rate is governed by a dimensionless number, known as the Stefan number (Ste), that is defined by:

$$
\text { Ste }=\left[C_{p}^{*}\left(T_{m}^{*}-T_{\infty}^{*}\right)\right] / \lambda,
$$

where $C_{p}^{*}$ is the specific heat of the material and $T_{m}^{*}$ is the temperature of the surroundings or some other appropriate reference temperature. The higher the value of Ste, the higher is the rate of the interface movement. Since Stefan's early investigation in 1891, several attempts have been made to capture all the phenomena that occur during melting and solidification of alloys and pure substances. We have already seen that change of phase may be accompanied by convection or the presence of a "mushy" region. Today vast numbers of application areas have emerged involving both natural as well as synthetic materials. Thus additional effects such as changes in properties (density, conductivity, specific heat), transfer of solutes in alloys, turbulence, surface tension at the free surface etc. must be considered along with multidimensionality and geometric complexities in the general problem of solidification and melting. Fortunately, the availability of computers allows consideration of all such effects in calculations intended to assist an engineer. 


\subsection{Some important applications}

There is hardly any product which, during its manufacture, does not undergo a process of solidification and melting at some stage. Casting, welding, soldering/ brazing, dip-forming, crystallisation etc. are typical manufacturing processes that involve melting and solidification. Processes such as rapid solidification, directional solidification or surface alloying are often used to create new materials or impart new physical and metallurgical properties to existing ones. The phenomenon known as "permafrost" is concerned with changes in load-bearing capacity of soils in very cold environments. The process of freezing and thawing is of interest in the preservation of foods. The principle of latent heat transfer is used in the development of compact thermal energy storage devices that enable storage and retrieval of energy at nearly constant temperature.

In all the above applications, it is important to know the rates of solidification and melting so that they can be controlled to meet the objectives at hand.

\subsection{The purpose of the review}

From the foregoing it is clear that solidification and melting are important phenomena in various applications. Being a transient phenomenon that takes place in impervious materials, experimental determination of melting and solidification rates through internal probing of the temperature field is a difficult task. Often the phenomenon takes place over very small regions, as for example in surface alloying. As such, experiments involve taking cross-sectional cuts to examine the microstructure of the material after subjection to different rates of solidification. The purpose of experimentation then is to draw general qualitative inferences; the experimentation itself being quite tedious.

It is for these reasons that solidification and melting phenomena have received considerable theoretical attention. Most of the earlier investigations were confined to one-dimensional diffusion-controlled problems. While one-dimensional problems provide useful guidance, multidimensional problems are of greater technological importance and are more difficult to solve. The availability of computers enabled the consideration of multidimensional phenomena involving convection as well as the presence of "mushy" region. In spite of the large number of publications dealing with solidification and melting, none of the methods used can be considered general enough to yield all quantities of interest (e.g., cooling rate, interface, temperature history etc.) under all types of boundary conditions.

The purpose of this review is to provide the most generalised formulation of melting and solidification problems and to examine the manner in which the formulation is particularised by different authors. Attention is directed to multidimensional problems involving numerical methods. The merits and demerits of different methods have been highlighted and the need for evolving a generalised method for solving melting and solidification problems has been emphasised.

\subsection{Outline of the paper}

In this section, melting and solidification problems are defined physically highlighting the important applications. In the second section, a generalised 
mathematical statement of any melting and solidification problem is derived along with source laws, boundary and initial conditions. Different ways of formulation are discussed and non-dimensional numbers governing the process are identified. Recent progress in numerical methods is reviewed in the third section, while the fourth section presents critical numerical aspects of all numerical methods. In the last section guidelines are provided for selecting numerical techniques. Areas needing emphasis in future research are also highlighted.

\section{Mathematical statement}

\subsection{Domain of interest}

Consider a region bounded by boundary $(B)$ and occupied by a pure substance (figure 1a). At some instant of time, the solid $(S)$ and liquid $(L)$ phases are separated by an interface $(I)$. The latent heat is released or absorbed at the interface which is at a fixed temperature, $T_{m}^{*}$. Due to the heat interaction at the boundary $(B)$, the interface moves through the domain with a velocity $v_{I}(x, y, z)$.

If the region is occupied by an impure substance (figure 1b), then the solid and liquid are separated by a finite "mushy" region that can be demarcated by $I_{s}$ (separating solid-solidus) and $I_{L}$ (separating liquid-liquidus). Both $I_{s}$ and $I_{L}$ move through the region as a result of heat interactions at the boundary. Every point in the region is defined by an orthogonal or non-orthogonal system. Our objective is to apply the conservation of energy, mass and momentum principles to small control volumes within each phase and at the interface.

When invoking the conservation of energy and mass principles, two approaches are possible-

(i) variable domain method; and

(ii) fixed domain method.

The essential difference between the two is that in the former the total domain is divided into two phases and the interface region; and each region is treated separately. Since the volume of each region changes with time, the method is termed as the variable domain method.

The fixed domain method does not deal with the particularised forms of the energy and mass conservation principle for each region. It rather considers the

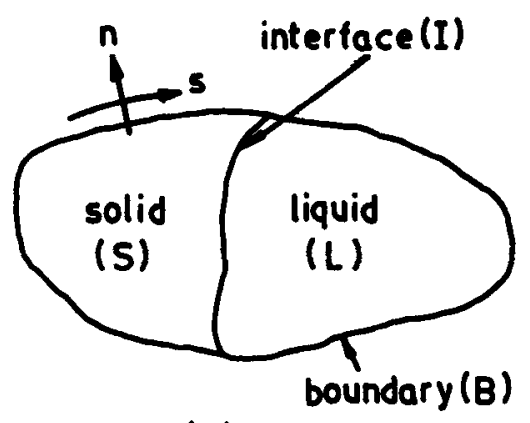

(a)

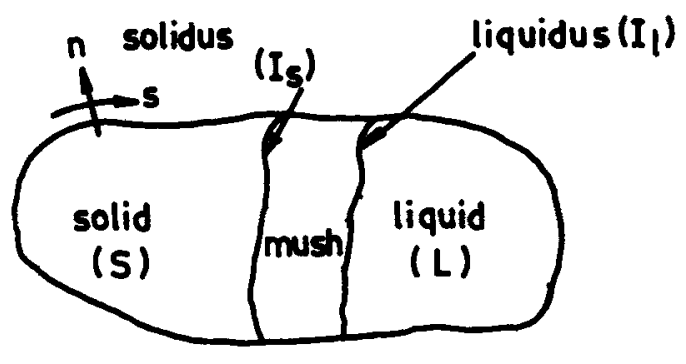

(b)

Figure 1. Domain of interest for (a) pure metal and (b) alloy. 
entire domain including all regions together; and thus since the total domain does not change with time, the method is termed as the fixed domain method.

Both methods have their advantages and disadvantages. It will be obvious though that in the variable domain method the interface location is explicitly identified a priori whereas in the fixed domain method the interface location is inferred from the solution of the governing equation.

\subsection{Variable domain method}

Solidification and melting being a thermo-fluid dynamic process, the application of the laws of conservation of mass, momentum and energy to control volumes situated in each phase yields partial differential equations governing the distribution of temperature $\left(T^{*}\right)$, concentration $\left(C^{*}\right)$, three velocity components $\left(u^{*}, v^{*}, w^{*}\right)$ and pressure $\left(p^{*}\right)$. The equations require information on material properties such as density $\left(\rho^{*}\right)$ and specific heat $\left(C_{p}^{*}\right)$ in addition to transport properties such as kinematic viscosity $\left(v^{*}\right)$, thermal diffusivity $\left(\alpha^{*}\right)$ and mass diffusivity $\left(D^{*}\right)$. The equations for any dependent variable can be written in generalised form as follows,

$$
\frac{\partial}{\partial t}\left(\rho^{*} \phi^{*}\right)+\operatorname{div}\left(\rho^{*} \mathbf{u}^{*} \phi^{*}\right)=\operatorname{div}\left(\Gamma^{*} \operatorname{grad} \phi^{*}\right)+S^{*}
$$

Table 1 provides the meanings of $\Gamma^{*}$ and $S^{*}$ for different $\phi^{\prime}$ s. The last mentioned entry in the table 1 (i.e., $\phi^{*}=1$ ) is the continuity equation applicable to a liquid phase. It enables determination of pressure distribution. In solid phase, of course, $\mathbf{u}^{*}=S_{u, v, w}^{*}=0$.

Since the properties can be assumed to be uniform for each phase, the equation can be non-dimensionalised. Thus defining,

$$
\begin{aligned}
& \theta=\frac{T^{*}-T_{m}^{*}}{T^{*}-T_{\times}^{*}}, \quad C=\frac{C^{*}-C_{s}^{* s}}{C_{l}^{* s}-C_{s}^{* s}}, \quad \mathbf{U}=\frac{\mathbf{u}^{*}}{U_{\mathrm{R}}}, \quad \mathbf{r}=\frac{\mathbf{r}^{*}}{L}, \\
& \rho=\frac{\rho^{*}}{\rho_{s}^{*}}, \quad k=\frac{k^{*}}{k_{s}^{*}}, \quad C_{p}=\frac{C_{p}^{*}}{C_{p s}^{*}}, \quad \mu=\frac{\mu^{*}}{\mu_{s}^{*}}, \\
& D=\frac{D^{*}}{D_{s}^{*}}, \quad p=\frac{p^{*}}{\rho_{s}^{*} U_{R}^{2}} \quad \text { and } \quad \tau=\frac{t \alpha_{s}^{*}}{L^{2}},
\end{aligned}
$$

where $L$ and $U_{R}$ are characteristic length and velocity and suffix $s$ refers to the solid phase, (2) can be written as,

$$
\frac{\partial(\rho \phi)}{\partial \tau}+\operatorname{Re} \cdot \operatorname{Pr} \cdot \operatorname{div}(\rho \mathbf{U} \phi)=\operatorname{div}(\Gamma \operatorname{grad} \phi)+S
$$

Table 1. Conservation equation.

\begin{tabular}{ccc}
\hline$\phi^{*}$ & $\Gamma^{*}$ & $S^{*}$ \\
\hline$u^{*}, v^{*}, w^{*}$ & $\mu^{*}=\rho^{*} v^{*}$ & $\left(\partial p^{*} / \partial n^{*}\right)+F_{b}^{*}$ \\
$T^{*}$ & $k^{*} / C_{p}^{*}=\rho^{*} \alpha^{*}$ & $\left(\phi_{\text {visc }}^{*}+R_{T}^{\prime \prime}\right) / C_{p}^{*}$ \\
$C^{*}$ & $\rho^{*} D^{*}$ & $R_{c^{*}}^{\prime \prime}$ \\
1 & 1 & 0 \\
\hline
\end{tabular}


Table 2. Non-dimensional conservation equation.

\begin{tabular}{ccc}
\hline$\phi$ & $\Gamma$ & $S$ \\
\hline$u, v, w$ & $\mu \operatorname{Pr}$ & $S_{u, v, w}^{*}\left\{L^{2} /\left(\rho_{s}^{*} \alpha_{s}^{*} U_{R}\right)\right\}$ \\
$\theta$ & $k / C_{p}$ & $S_{\theta}^{*}\left\{L^{2} /\left[\rho_{s}^{*} \alpha_{s}^{*}\left(T_{m}^{*}-T_{\infty}^{*}\right)\right]\right\}$ \\
$C$ & $\rho D(\operatorname{Pr} / \mathrm{Sc})$ & $S_{c}^{*}\left\{L^{2} /\left[\rho_{s}^{*} \alpha_{s}^{*}\left(C_{l}^{* s}-C_{s}^{* s}\right)\right]\right\}$ \\
1 & 1 & 0 \\
\hline
\end{tabular}

where $\phi, \Gamma$ and $S$ are defined as in table 2 .

The different non-dimensional numbers mentioned in table 2 and (3) are defined as follows,

$$
\begin{aligned}
& \operatorname{Re}=\rho_{s}^{*} U_{R} L / \mu_{s}^{*}=U_{R} L / v_{s}^{*}, \\
& \operatorname{Pr}=C_{r}^{*} \mu_{s}^{*} / k_{s}^{*}=\mu_{s}^{*} /\left(\rho_{s}^{*} \alpha_{s}^{*}\right)=v_{s}^{*} / \alpha_{s}^{*}, \\
& \mathrm{Sc}=\frac{\mu_{s}^{*}}{\rho_{s}^{*} D_{s}^{*}}=\frac{v_{s}^{*}}{D_{s}^{*}} .
\end{aligned}
$$

Note that in the solid phase $\rho, D, k, C$ and $\mu$ take the value of unity.

\subsection{Boundary and initial conditions}

Solidification and melting being a transient phenomenon, initial conditions must be specified for solution of equations along with the boundary conditions. Thus at the commencement of melting of a solid, for example, the velocities are zero and the concentration and temperature can be assumed to be uniform and known. The initial temperature may equal the environmental temperature $T_{\infty}^{*}$ or some other temperature including $T_{m}^{*}$. At the beginning of the solidification, however, the initial conditions must be those at the end of melting. Thus the velocities in the liquid phase may be finite and temperature and concentration may be non-uniform with the temperature in the liquid phase often exceeding $T_{m}^{*}$.

The boundary conditions, however, require careful consideration in different applications. We shall, therefore, consider each variable separately.

2.3a Temperature boundary condition: The most frequently obtained boundary condition for temperature is that of an energy flux, $q_{b}^{*}$. Either the flux is specified at the boundary or it must be inferred from the temperature of the boundary, $T_{b}^{*}$ and its environment, $T_{\infty}^{*}$. Thus for heating or cooling flux,

$$
-\left.k_{s, l}^{*} \frac{\partial T^{*}}{\partial n^{*}}\right|_{b}=q_{b}^{*}(s)=h^{*}(s)\left(T_{b}^{*}-T_{\infty}^{*}\right)+i_{R} \sigma\left(T_{b}^{* 4}-T_{\infty}^{* 4}\right),
$$

where $s$ is the distance along the boundary.

In surface melting, welding etc, $q_{b}^{*}(s)$ is known as the heating flux over a part of the boundary, and for the remainder of the boundary $h^{*}(s)$ and $\varepsilon_{R}$ must be known. Often $q_{b}^{*}(s)$ has a specified (Gaussian or top-hat) distribution. In applications such as crystal growth, heat energy storage etc., $q_{b}^{*}(s)$ is not specified but energy transfer takes place by convection and radiation (e.g., crystal growth, permafrost) or by convection alone (e.g., heat storage devices). In the manufacture of turbine blades by 
unidirectional solidification, radiant heat transfer is the only mechanism used. Often zero flux boundary conditions can be employed on a part of the boundary where adiabatic conditions prevail (e.g. symmetry planes and insulated surfaces).

In terms of the dimensionless parameters employed earlier, (4) can be written as,

$$
-\left.k \frac{\partial \theta}{\partial n}\right|_{b}=q_{b}(s)=\left(\mathrm{Bi}+R_{F}\right) \theta_{b},
$$

where

$$
q_{b}(s)=q_{b}^{*}(s) /\left[\left(k_{s}^{*} / L\right)\left(T_{m}^{*}-T_{\infty}^{*}\right)\right]
$$

2.3b Concentration boundary condition: Usually the boundary condition for concentration is one of zero mass flux, e.g.,

$$
\left.\frac{\partial C}{\partial n}\right|_{b}=0
$$

However, in surface alloying where alloying elements are added to a part of the molten metal, the condition is,

$$
-\left.\mathrm{D} \frac{\partial C}{\partial n}\right|_{b}=j_{b}(s) .
$$

2.3c Velocity boundary condition: When the liquid phase is bounded by solid walls, the boundary condition is one of no-slip, e.g.,

$$
u^{*}=v^{*}=w^{*}=0 \cdot 0 \text {. }
$$

However, a part of the boundary may often be a free surface and convection is driven by surface tension forces that result from the temperature gradients. Under such circumstances, the condition can be derived with reference to the figure 2 where force balance between fluid shear and surface tension is demonstrated in two

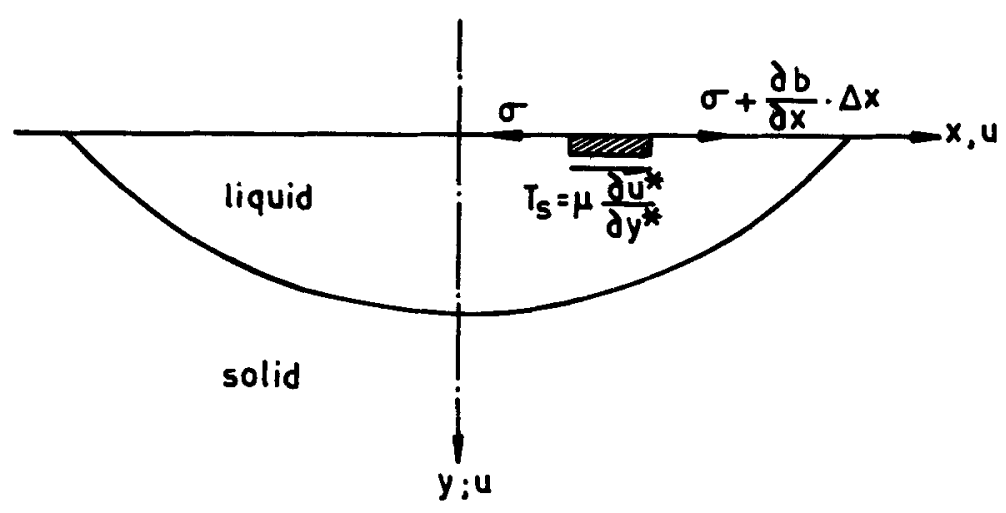

Figure 2. Force balance at the frec surface of the molten pool. 
dimensions. Thus,

$$
\left.\mu_{l}^{*} \frac{\partial u^{*}}{\partial y^{*}}\right|_{b}=-\frac{\partial \sigma_{s}^{*}}{\partial x^{*}}=-\left.\left(\frac{\mathrm{d} \sigma_{s}^{*}}{\mathrm{~d} T^{*}}\right) \frac{\partial T^{*}}{\partial x^{*}}\right|_{b},
$$

or in dimensionless form,

$$
\left.\mu_{l} \frac{\partial u}{\partial y}\right|_{b}=-\left.R_{S F} \frac{\partial \theta}{\partial x}\right|_{b}
$$

For the other velocities, we note that,

$$
\left.\mu_{l} \frac{\partial w}{\partial y}\right|_{b}=-\left.R_{S F} \frac{\partial \theta}{\partial z}\right|_{b}
$$

and

$$
v=0 \cdot 0 \text {. }
$$

\subsection{Source laws}

In tables 1 and 2, sources (or sinks) have been identified for each variable of interest. We consider the variables in turn.

2.4a Momentum sources: For velocities $u^{*}, v^{*}, w^{*}$, the dimensional source term is,

$$
S^{*}=\left(\partial p^{*} / \partial n^{*}\right)+F_{b}^{*}
$$

Writing the obvious pressure gradient term $\left(\partial p^{*} / \partial n^{*}\right)$ in dimensionless form yields,

$$
S=\operatorname{Re} \operatorname{Pr} \partial p / \partial n+F_{b}^{*} L^{2} /\left(\rho_{s}^{*} U_{R} \alpha_{s}^{*}\right) .
$$

Two types of convection generating body forces $F_{b}^{*}$ are common,

i) buoyancy forces; and

ii) electromagnetic forces.

The first type of force is commonly known to arise as a result of density differences acting against the gravity force, the density differences being caused by temperature or concentration differences. Under these conditions,

$$
F_{b}^{*} \text { (buoyancy) }=\rho_{l}^{*} \beta^{*} g\left(\phi^{*}-\phi_{\mathrm{ref}}^{*}\right),
$$

where $\phi^{*}$ represents $T^{*}$ or $C^{*}$ and $\phi_{\mathrm{ref}}^{*}$ is $T_{m}^{*}$ in thermal buoyancy and $C_{l}^{* s}$ in concentration buoyancy. Similarly $\beta^{*}$ equals

$$
-\frac{1}{\rho_{l}^{*}}\left(\frac{\partial \rho_{l}^{*}}{\partial T^{*}}\right)_{p^{*}} \text { or }-\frac{1}{\rho_{l}^{*}}\left(\frac{\partial \rho_{l}^{*}}{\partial C^{*}}\right)_{p^{*}} \text { respectively. }
$$

The electromagnetic force which arises due to electron beam heating is expressed in vector form as,

$$
F_{b}^{*}(\text { electromagnetic })=-\mu_{m}^{*} H_{m}^{*} \times\left(\nabla \times H_{m}^{*}\right)
$$


Now normalising $H^{*}$ by $H_{0}$, the total source term can be written as,

$$
\begin{aligned}
S & =\operatorname{Re} \operatorname{Pr} \frac{\partial p}{\partial n}+\left(\frac{\rho_{l}}{\rho_{s}}\right) \frac{\mathrm{Gr}_{h} \operatorname{Pr}}{\operatorname{Re}} \theta+\left(\frac{\rho_{l}}{\rho_{s}}\right) \frac{\mathrm{Gr}_{m} \operatorname{Pr}}{\operatorname{Re}} C \\
& +\frac{\operatorname{Re} \operatorname{Pr}}{M_{m}^{2}}\left[H_{m} \times\left(\nabla \times H_{m}\right)\right] .
\end{aligned}
$$

2.4b Mass and energy sources: Solidification and melting are rarely accompanied by a chemical reaction. Thus sources of mass and energy equations are absent. The melting process may however be brought about by induction heating and a finite volumetric heat generation rate must be considered in each phase in such a case.

\subsection{Interface conditions}

In most applications the interface movement rate is sufficiently low for equilibrium conditions to prevail at the interface. Thus two types of conditions are invoked at the interface,

(i) equilibrium condition,

(ii) energy and mass flux condition.

The no-slip condition for velocities is of course satisfied.

2.5a Equilibrium conditions: For pure substances, the interface is an isothermal surface with temperature equal to $T_{m}^{*}$. For an alloy, on the other hand, the liquidus temperature is governed by the solute concentration and can be determined from the phase diagram. Often the interface turns out to be a curved surface and the magnitude of the saturation temperature is known to depend on the surface tension $\left(\sigma_{s}\right)$, the entropy of melting $(s)$ and the local surface curvature $(R)$. Thus three possibilities arise which can be expressed in non-dimensional form as follows,

$$
\left.\begin{array}{rl}
\theta_{I}= & 0 \cdot 0, \quad \text { (for pure metals), } \\
= & m\left\{\left[\left(C_{l}^{* s}-C_{s}^{* s}\right) /\left(T_{m}^{*}-T_{\infty}^{*}\right)\right] C+\left[C_{s}^{* s} /\left(T_{m}^{*}-T_{\infty}^{*}\right)\right]\right\}, \\
& (\text { for alloys), } \\
= & -\left(\sigma_{s} / s L\right)\left[1 / R\left(T_{m}^{*}-T_{\infty}^{*}\right)\right], \quad \text { (for curved interfaces). }
\end{array}\right\}
$$

2.5b Flux condition for pure substances: A separate energy balance is required at the interface because of release or absorption of latent heat during solidification or melting, respectively. The energy balance is as follows,

$$
\left(k^{*} \frac{\partial T^{*}}{\partial n^{*}}\right)_{l}-\left(k^{*} \frac{\partial T^{*}}{\partial n^{*}}\right)_{s}=-\rho^{*} \lambda v_{l}^{*} .
$$

Normalising this equation (as mentioned in $\S 2.2$ ), the following form is obtained

$$
\left(k \frac{\partial \theta}{\partial n}\right)_{l}-\left(k \frac{\partial \theta}{\partial n}\right)_{s}=-(\operatorname{Re} \operatorname{Pr} / \text { Ste }) \rho v_{I} .
$$


The significance of the dimensionless number, Ste, is now clear from (20), e.g. the higher the Ste the faster is the rate of interface movement. The values of Ste for different important applications are given in table 3.

2.5c Flux condition for alloyed substances: For alloys, both energy and mass balance are required across the mushy region. Energy balance, e.g., the heat flux condition, is the same as for the pure substances, (20). Because of the jump in the solute concentration between liquidus and solidus, a separate mass balance is needed across the mushy region. Thus the mass balance condition yields,

$$
\left(D^{*} \frac{\partial C^{*}}{\partial n^{*}}\right)_{l}-\left(D^{*} \frac{\partial C^{*}}{\partial n^{*}}\right)_{s}=-\left(C_{l}^{*}-C_{s}^{*}\right) v_{l}^{*}
$$

Normalising this equation, one obtains

$$
\left(D \frac{\partial C}{\partial n}\right)_{l}-\left(D \frac{\partial C}{\partial n}\right)_{s}=-\frac{\operatorname{Re} \operatorname{Pr}}{\operatorname{Le}} v_{I}
$$

where $\mathrm{Le}=$ Lewis number $=\operatorname{Pr} / \mathrm{Sc}$.

Note that the speed of the interface movement is dependent on both mass and heat diffusion [e.g., (20) and (22)] in the case of alloy solidification problems. Hence, both Ste and Le have to be high for faster interface movement.

\subsection{Summary}

The phase change process is thus governed by several dimensionless parameters. The transfer processes within each phase are governed by $\mathrm{Re}, \operatorname{Pr}$ and Sc. The boundary conditions give rise to $\mathrm{Bi}, R_{F}$ and $R_{S F}$ representing the effects of external convection, radiation and surface tension. The source laws yield $\mathrm{Gr}_{n}, \mathrm{Gr}_{m}$ and $M_{m}$, representing effects of buoyancy due to temperature and concentration differences, and of magnetic field, respectively.

The interface and mushy region processes give rise to Ste and Le, the former being the result of energy transfer and the latter of mass diffusion.

The rate of solidification and melting in the most general problem is thus governed by

Phase change rate $=F+\operatorname{Re}, \mathrm{Pr}, \mathrm{Sc}, \mathrm{Gr}_{n}, \mathrm{Gr}_{m}, M_{m}, \mathrm{Bi}, R_{F}, R_{S F}$, Ste, Le, . . othersł,

Table 3. Typical values of Stefan numbers.

\begin{tabular}{lc}
\hline Application & Ste \\
\hline Casting and processing of pure metals with large temperature range & 1 to 3 \\
Casting and processing of alloys with large temperature range & 0.2 to 0.3 \\
Thermal energy storage, freezing and melting of water by nature, both within small & 0.0 to 0.2 \\
temperature range. & \\
\hline
\end{tabular}

Reference: Shamsunder (1978). 
where $F(\quad)$ means "function of" and other symbols signify property ratios between phases, geometry, interface curvature and the properties of the phase diagram. The fixed domain method will now be considered.

\subsection{Fixed domain method}

2.7a The energy equation: In the variable domain method the transport of sensible heat in the two phases and that of latent heat at the interface were treated separately; these transport rates however were balanced by the conduction heat transfer rates (and sources if any). Both the sensible heat as well as the latent heat are different forms of energy and the changes in them contribute to changes in enthalpy. Use of enthalpy, rather than the temperature, thus offers an opportunity for treating the two phases as well as the interface in a unified way. Thus irrespective of the region being considered, the energy transfer is governed by the following equation

$$
\frac{\partial}{\partial t}\left(\rho^{*} H^{*}\right)+\operatorname{div}\left(\rho^{*} \mathbf{u}^{*} H\right)=\operatorname{div}\left(k^{*} \operatorname{grad} T^{*}\right)
$$

We however now have an equation, which has two dependent variables: enthalpy $H^{*}$ and temperature $T^{*}$. We thus need a relationship between $H^{*}$ and $T^{*}$, in order that (23) can be solved for the entire domain without specific reference to the phases and the interface region. The fixed domain method, with use of enthalpy, is designed to take advantage of this fact.

Figures $3 \mathrm{a}$ and $\mathrm{b}$ show the typical relationship between $H^{*}$ and $T^{*}$ for the pure substance and an alloy. In the completely solid and the liquid regions, the relationship between $H^{*}$ and $T^{*}$ is simply linear; the difficulty however arises at the interface for a pure metal and in the mushy region for an alloy.

Different authors have made proposals for the $H^{*}-T^{*}$ relationship particularly

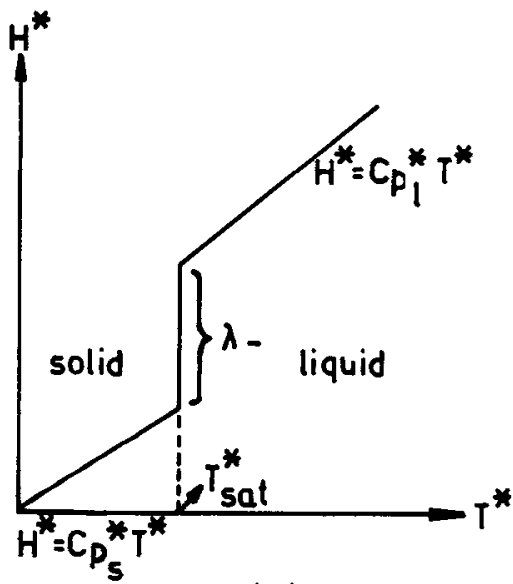

(a)

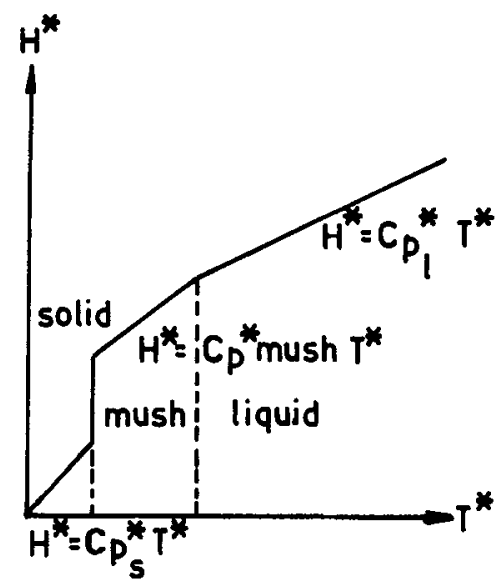

(b)

Figure 3. Enthalpy $\left(H^{*}\right)$ and temperature $\left(T^{*}\right)$ relationships for (a) pure metal and (b) alloy. 
for the case of a pure metal. Our purpose is to seek similarities, or otherwise, between them and to examine their consequences in the calculation of the time derivative $i H^{*} / \hat{\imath} t$ at the interface and within each phase.

Pure metals-- Thus, all models use the following relationships for the solid and the liquid regions:

$$
\begin{aligned}
& \text { Solid: } \quad H^{*}=C_{p s}^{*} T^{*}, \text { for } T^{*}<T_{m}^{*}, \\
& \text { Liquid: } \quad H^{*}=C_{p l}^{*} T^{*}, \text { for } T^{*}>T_{m}^{*} .
\end{aligned}
$$

For the interface where $T^{*}=T_{m}^{*}$, however, there are four proposals which we shall refer to as models. Thus,

Model 1-Szekely \& Themlis (1970)

$$
H_{I}^{*}=f\left(C_{p}^{*} T^{*}\right)
$$

where $f$ is a continuous linear function of $T^{*}$ which is assumed to vary over a small temperature interval $2 \varepsilon$ or

$$
T_{m}^{*}-\varepsilon<T^{*}<T_{m}^{*}+\varepsilon
$$

Model 2-Meyer (1971),

$$
\begin{aligned}
H_{I}^{*} & =C_{p s}^{*} T^{*}+(\lambda / 2 \varepsilon)\left(T^{*}-T_{m}^{*}+\varepsilon\right) \\
& =C_{P_{e S S}}^{*} T^{*}+(\lambda / 2 \varepsilon)\left(\varepsilon-T_{m}^{*}\right),
\end{aligned}
$$

with $T_{m}^{*}-\varepsilon<T^{*}<T_{m}^{*}+\varepsilon$,

and $C_{p_{(\prime \prime)}}^{*}=C_{p s}^{*}+(\lambda / 2 \varepsilon)$.

Model 3 - Bonacina et al (1975),

$$
H_{I}^{*}=C_{P_{e f f}}^{*} T^{*}
$$

with $T_{m}^{*}-\varepsilon<T<T_{m}^{*}+\varepsilon$, and $C_{p_{(j)}}^{*}=\lambda / 2 \varepsilon$.

Model 4-Shamsundar \& Sparrow (1975a),

$$
\left(1 / V^{*}\right) \int_{V^{*}} H_{I}^{*} \mathrm{~d} V^{*}=\left(1 / V^{*}\right) \int_{V^{*}}\left(C_{p}^{*} T_{m}^{*}+\lambda\right) \mathrm{d} V^{*}
$$

The first three models simply assume that melting takes place over a range of temperatures rather than at a fixed value of the temperature i.e. $T_{m}^{*}$. Model 1 and model 3 are similar, except that the latter assumes linearity between $H^{*}$ and $T^{*}$, whereas the former allows for a nonlinear variation. Model 2 is similar to model 3 , but the linearity constant is different. Figure 4 shows graphical representation of the three models. Note that since $\varepsilon$ is very small, the variation of $C_{p}$ in model 3 shows a very sharp and spike-like variation.

Unlike the first three models, model 4 assumes that melting takes place over a small control volume $V^{*}$ rather than over a small range of temperature.

The time-derivative of enthalpy then takes the following forms,

$$
\text { Solid } \quad \frac{\partial H^{*}}{\partial t}=\frac{\partial}{\partial t}\left(C_{p s}^{*} T^{*}\right)
$$




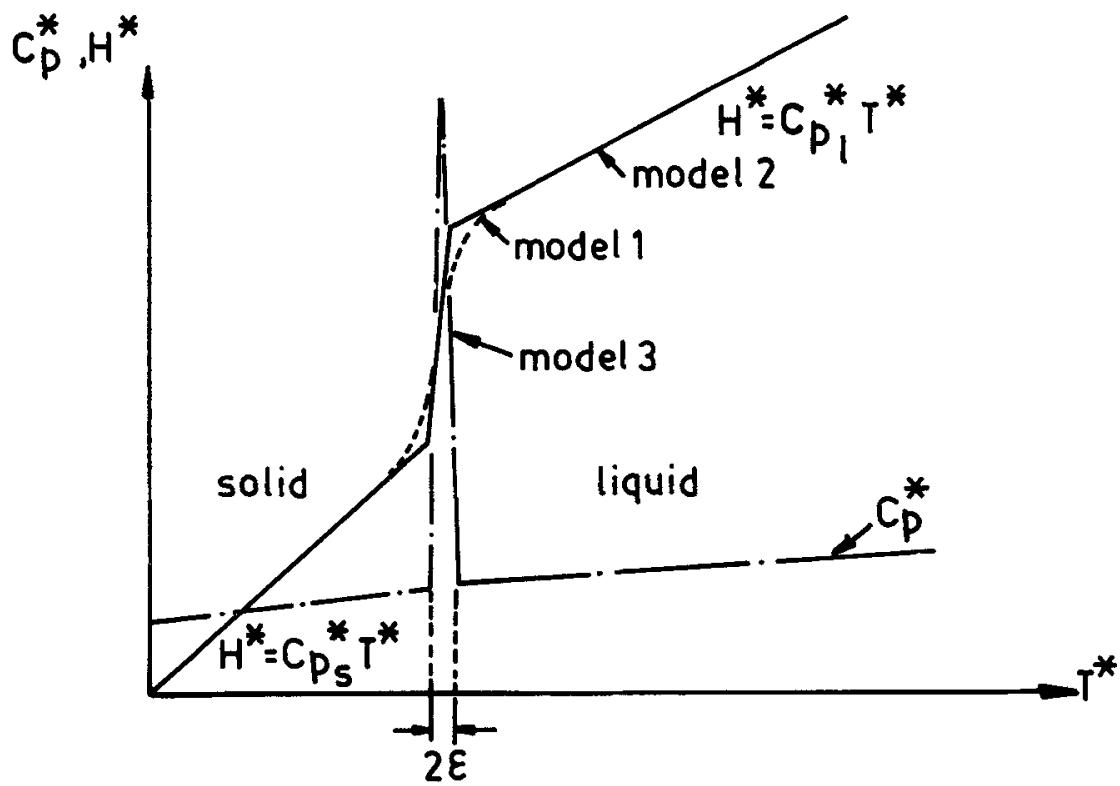

Figure 4. Approximation of $H^{*} T^{*}$ and $C_{p}-T^{*}$ relationships.

Liquid $\quad \frac{\partial H^{*}}{\partial t}=\frac{\partial}{\partial t}\left(C_{p l}^{*} T^{*}+\lambda\right)=\frac{\partial}{\partial t}\left(C_{p l}^{*} T^{*}\right)$

Interface

Model $\left.1 \quad \frac{\partial H^{*}}{\partial t}\right|_{I}=\frac{\partial f}{\partial T^{*}} \cdot \frac{\partial T^{*}}{\partial t} ;$

Models $\left.2 \& 3 \frac{\partial H^{*}}{\partial t}\right|_{I}=\frac{\partial}{\partial t}\left(C_{p_{e f f}}^{*} T^{*}\right)$

Model $4 \quad \frac{1}{V^{*}} \frac{\partial}{\partial t} \int_{V^{*}} H^{*} \mathrm{~d} V^{*}=\frac{1}{V^{*}} \int_{V^{*}} \frac{\partial H^{*}}{\partial t} \mathrm{~d} V^{*}+\frac{\lambda}{V^{*}} \frac{\partial V^{*}}{\partial t}$

$$
\begin{aligned}
& =\frac{1}{V^{*}} \int_{V^{*}} \frac{\partial H^{*}}{\partial t} \mathrm{~d} V^{*}+\lambda \frac{\partial f_{l}}{\partial t} \\
& =\frac{1}{V^{*}} \int_{V^{*}} \frac{\partial}{\partial t}\left(C_{p}^{*} T_{m}^{*}\right) \mathrm{d} V^{*}+\lambda \frac{\partial f_{l}}{\partial t},
\end{aligned}
$$

where (34) \& (35) result from Leibniz's rule and $f_{l}$ is the liquid fraction.

Models 1, 2 and 3 thus allow replacement of $H^{*}$ in (23) by $T^{*}$ with proper values being assigned to $\partial f / \partial T^{*}$ and $C_{p_{\text {eff }}}$. Equation (23) can now be solved by any discretization technique without specific reference to interface. The new empirical inputs however are the values of $\varepsilon$ and the $\partial f / \partial T^{*}$ function.

An additional point to note, however, is that models 2 and 4 can also be 
interpreted in another way. If it is argued that melting takes place not only over a finite temperature range or a finite volume, but also over a finite time $\Delta t$ (say), then a relationship between $H^{*}$ and $T^{*}$ for all regions can be written as,

$$
H^{*}(x, y, z, t)=C_{p}^{*} T^{*}(x, y, z, t)+H_{p s}^{*}(t)
$$

such that

$$
\int_{t}^{t+\Delta t} \frac{\mathrm{d} H_{p s}^{*}}{\mathrm{~d} t} \cdot \mathrm{d} t=\lambda .
$$

The meaning of $H_{p s}^{*}(t)$ can now be interpreted as:

Model $2-H_{p s}^{*}(t)=(\lambda / 2 \varepsilon)\left(T^{*}-T_{m}^{*}+\varepsilon\right)$;

Model $4-\frac{\mathrm{d} H_{p s}^{*}(t)}{\mathrm{d} t}=\lambda \frac{\mathrm{d} f l}{\mathrm{~d} t}$.

Equations (38) and (39) will be referred as models $2 \mathrm{a}$ and $4 \mathrm{a}$, respectively, in the rest of this paper. If (36) is used then replacement of $H^{*}$ will yield a source term in the energy equation i.e. $\mathrm{d} H_{p s}^{*} / \mathrm{d} t$; and again the equation can be solved without specifically locating the interface; care is however needed in the choice of $\Delta t$ when numerical solution over discrete time steps is sought, so that (37) is satisfied. It is quite clear then, that in model 2 correct choice of $\Delta t$ and $\varepsilon$ must be made simultaneously; any arbitrariness in the choice of $\varepsilon$ must result in an erroneous solution.

The models presented above are, of course, deceptively simple when numerical solutions are sought over discrete time and space intervals. Although convenient to use, it is often found that the enthalpy models provide solutions that are at variance with exact solutions (available for a 1D case) or expectations.

Alloys-For alloys, the $H^{*}-T^{*}$ relationships used are (see figure $3 \mathrm{~b}$ ).

$$
H^{*}=\left\{\begin{array}{lll}
C_{p_{s}}^{*} T^{*} & ; & T^{*}<T_{E}^{*} \\
C_{p_{s}}^{*} T^{*}+f_{l E} \cdot \lambda & ; & T^{*}=T_{E}^{*} \\
C_{p_{\text {mush }}}^{*} T^{*}+f_{l} \lambda & ; & T_{E}^{*}<T^{*} \leqslant T_{l}^{*} \\
C_{p_{l}}^{*} T^{*}+\lambda & ; & T^{*}>T_{l}^{*}
\end{array}\right\},
$$

where $C_{p_{\text {mush }}}^{*}=f_{l} \cdot C_{p_{l}}^{*}+\left(1-f_{l}\right) C_{p_{s}}^{*}$,

$f_{l E}$ is the eutectic liquid fraction and $f_{l}$ is the liquid fraction, determined from the mass diffusion considerations (see $\S 2.7 \mathrm{~b}$ ).

It will be noticed that unlike pure metals, melting and solidification of alloys truly takes place over a range of temperatures, e.g., $T_{E}^{*} \leqslant T^{*} \leqslant T_{l}^{*}$ and models 1 , 2 and 4 can be directly applied by noting that $2 \varepsilon=T_{l}^{*}-T_{E}^{*}$.

2.7b Mass transfer equation: The concept of fixed domain formulation can also be extended to the mass transfer equations. In the mass transfer problem there is a jump in the concentration profile, e.g. between $C_{l}^{* s}$ (liquidus) and $C_{s}^{* s}$ (solidus) (figure 5) across the mushy region. As a result, the mass diffusion term will be divergent across the mushy region. The fixed domain formulation, thus needs an alternative form of mass transfer equation with a different variable (which is 


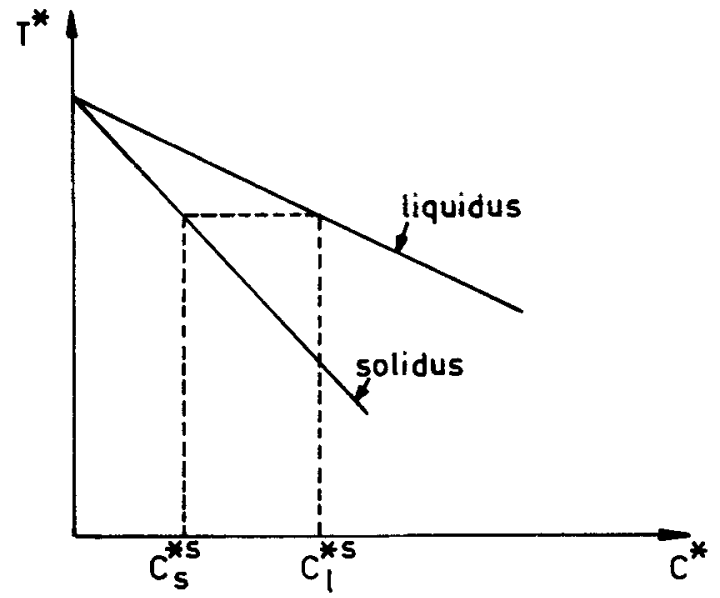

Figure 5. A simplified phase diagram.

continuous across the mushy region) for evaluating the mass diffusion term. From the equilibrium diagram, it can be seen that,

$$
\left.\begin{array}{l}
C_{l}^{* s}=m_{l} T_{l}^{*} \\
C_{s}^{* s}=m_{s} T_{s}^{*}
\end{array}\right\}
$$

If $m$ is defined as

$$
m=\left\{\begin{array}{l}
m_{l} \\
m_{s}
\end{array}\right.
$$

we can see the $C / m$ is continuous across the mushy region. On this basis, Fix (1978) defined a variable $G^{*}$ as,

$$
G^{*} \equiv C^{*} / m \text {. }
$$

The modified form of mass conservation equation is then as follows

$$
\partial C^{*} / \partial t+\operatorname{div}\left(\mathbf{u}^{*} \cdot C\right)=\operatorname{div}\left(m D^{*} \operatorname{grad} G^{*}\right) .
$$

Equation (45) thus represents the mass conservation in the whole domain. The different properties and the concentration jump across the mushy region are thus bound up in the functional form of $C^{*}\left(G^{*}\right)$ which is as follows,

$$
C^{*}=\left\{\begin{array}{l}
m_{s} G^{*} \text { in the solid, } \\
\text { between } m_{s} G^{*} \& m_{l} G^{*} \text { in the mushy region, } \\
m_{l} G \text { in the liquid. }
\end{array}\right.
$$

Another approach to include mass transfer is to write an integral mass balance across the mushy region and thus determine the amount of material which has undergone the phase change (e.g., $f_{l}$ ). Once the $f_{l}$ is known it can be directly included in model 4 to calculate enthalpy correctly. Depending on the assumption, a series of expressions are available in the literature to determine $f_{l}$. All these expressions are based on the well-mixed liquid assumption, e.g., there is no concentration gradient in the liquid. The rate of mass diffusion in liquid alloys is very slow compared to thermal diffusion-for example $D^{*}$ is $10^{-7}$ to $10^{-8} \mathrm{~m}^{2} / \mathrm{s}$ for 
$\mathrm{Mn}$ in steel (Chande \& Mazumder 1985), where $\alpha^{*}$ is $10^{-5}$ to $10^{-6} \mathrm{~m}^{2} / \mathrm{s}$. As a result, mass transfer is important in the mushy region, solid phase and liquid phase. The solute boundary layer, on the liquid-side is very thin compared to the thermal boundary layer and hence a well-mixed condition is a reasonable assumption for liquid alloys.

The various integral mass balance equations are as follows,

Scheil equation (Flemings 1974, pp. 160-163)-

$$
f_{l}=\left[\left(T_{m}^{*}-T^{*}\right) /\left(T_{m}^{*}-T_{l}^{*}\right)\right]^{-1 /\left(1-k^{\prime}\right)} .
$$

Brody-Fleming's equation (Brody \& Flemings 1966)-

$$
f_{l}=\left[1 /\left(1-2 X_{b} k^{\prime}\right)\right]\left[\left(T_{m}^{*}-T^{*}\right) /\left(T_{m}^{*}-T_{l}^{*}\right)\right]^{-\left(1-2 X_{b} k^{\prime}\right) /\left(1-k^{\prime}\right)} .
$$

Clyne and Kurz's equation (Clyne \& Kurz 1981),

$$
\left.f_{l}=\left[1 /\left(1-2 X_{c} k^{\prime}\right)\right]\left[T_{m}^{*}-T^{*}\right) /\left(T_{m}^{*}-T_{l}^{*}\right)\right]^{-\left(1-2 X_{c} k^{\prime}\right) /\left(1-k^{\prime}\right)} .
$$

Here, $k^{\prime}$ is the partition coefficient and is defined as

$$
k^{\prime}=C_{l}^{* s} / C_{s}^{* s} \text {. }
$$

Equation (47) assumes that the diffusion of solute in solid is negligible. For slow rates of solute diffusion in solids, (28) is used whereas for faster rates (49) is used. $X_{b}$ is known as the "Brody-Flemings back diffusion" parameter and defined as

$$
X_{b}=4 D_{s}^{*} t_{f} / L^{2},
$$

where $t_{f}$ is the local solidification time. $X_{c}$, the modified back diffusion parameter, is related to $X_{b}$ as

$$
X_{c}=X_{b}\left[1-\exp \left(-1 / X_{b}\right)\right]-\frac{1}{2} \exp \left(-1 / 2 X_{b}\right) .
$$

2.7c Momentum equation: The usual way of solving a momentum equation along with the enthalpy formulation is to solve the equations only in the liquid control volumes. But the advantage of the fixed domain formulation is lost because one has to identify the liquid control volumes, e.g., the interface position is needed. The papers of Chan et al $(1983$, pp. 150-157, 1984, 1985) are of this type. There are two ways of extending the fixed domain concept to the solution of momentum equations - the variable viscosity model and the porous media model.

Variable viscosity model-In this model, viscosity, $\mu^{*}$, is assumed to be a function of temperature such that in the phase change and solid region $\mu^{*}$ takes a high value. The function should be dependent on the latent heat change of a control volume and thus it is proper to assign an approximate pseudo-viscosity depending on the amount of liquid in the control volume. The variable viscosity is thus defined as follows (Voller et al 1987),

$$
\mu^{*}=\mu_{l}^{*}+B_{M}\left[\lambda-H_{p s}^{*}(t)\right],
$$

where $H_{p s}^{*}(t)$; introduced in (36) and (37), is the representative latent heat of the control volume and $B_{M}$ is some large value. In the liquid $H_{p s}^{*}(t)=\lambda$ and hence 
$\mu^{*}=\mu_{l}^{*}$. in the solid region takes a high value, e.g.

$$
\mu_{s}^{*}=\mu_{l}^{*}+B_{M} \lambda
$$

and thus ensures zero velocity in that region. Depending upon the value of $H_{p s}^{*}(t), \mu^{*}$ takes different values in the phase change region. A typical $\mu^{*}-T^{*}$ relationship is shown in figure 6.

Equation (51) is suitable for pure metals. For alloys, where the actual value of $f_{l}$ is known from the mass balance, the suitable definition of viscosity is as follows

$$
\mu^{*}=\mu_{l}^{*}, f_{l}+B_{M}\left(1-f_{l}\right),
$$

This relationship also ensures a large value of $\mu^{*}$ in the solid, while $\mu^{*}$ in the mushy region is dependent on $f_{l}$.

Porous media model-This model is relevant for alloy solidification problems and treats the mushy region as a porous medium. The mushy region consists of dendrites with primary, secondary or other arms and nearly saturated liquid. As a result, flow in the mushy region can be treated as a liquid fraction flowing through a solid matrix. This model was first suggested by Mehrabian et al (1970) while analysing the effect of indendritic fluid flow on microsegregation.

The flow through a porous medium is governed by the well-known Darcy's law. The law states that velocity of flow in a porous medium is proportional to the pressure gradient. e.g.,

$$
u^{*}=-k^{*}\left(\hat{c} p^{*} / \partial x^{*}\right)
$$

Hence, the flow in the porous medium can be accounted for by the momentum equation (3) replacing source $S^{*}$ by $-u^{*} / k^{*}$. The permeability, $k^{*}$, in the mushy region is directly related to the fraction of liquid present. As $f_{l} \rightarrow 0$, the permeability $k^{*} \rightarrow 0$ and the source term (which is of high value) will dominate and thus force the related velocity close to zero. A suitable choice for the source term then will be

$$
S^{*}=B_{M}\left(1-f_{l}\right)
$$

Except the linear Darcy source (54), a nonlinear Darcy source term can also be used (Voller et al 1987).

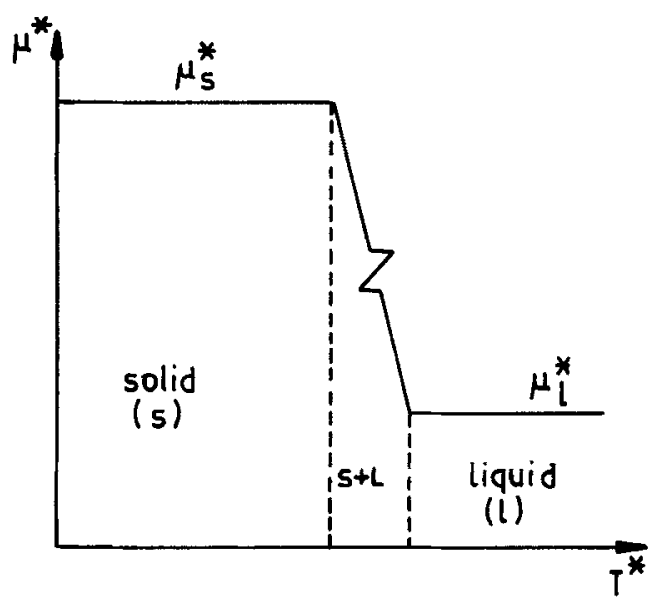

Figure 6. A typical $\iota^{*} T^{*}$ relationship 


$$
S^{*}=B_{M}\left[\exp \left(1-f_{l}\right)-1\right] \text {. }
$$

Different sources used by Voller et al (1987), are as follows,

Viscosity (arithmetic), $B_{M}=200$,

Viscosity (harmonic), $B_{M}=200$,

Linear Darcy source, $B_{M}=200$,

Nonlinear. Darcy source, $B_{M}=6.78$.

\subsection{Normalisation of fixed domain equation}

The fixed domain equations can now be normalised in the following way,

$$
\begin{aligned}
& \psi=\left(H^{*}-H_{\mathrm{sat}}^{*}\right) / \lambda, \quad C=\left(C^{*}-C_{s}^{* s}\right) /\left(C_{l}^{* s}-C_{s}^{* s}\right), \\
& \left.\theta=C_{p}^{*}\left(T^{*}-T_{m}\right) / \lambda, \quad G=m_{s}^{*}\left(G^{*}-G_{m}^{*}\right) / C_{l}^{* s}-C_{s}^{* s}\right) .
\end{aligned}
$$

Other variables (e.g. $u^{*}, \rho^{*}, C_{p}^{*}, \alpha^{*}, p^{*}, t^{*},$. ) are normalised as mentioned in $\$ 2.2$. The generalised form of the non-dimensional governing equation is expressed as follows

$$
a \frac{\partial\left(b \phi_{t}\right)}{\partial t}+\operatorname{Re} \cdot \operatorname{Pr} \operatorname{div}\left(\rho \mathbf{u} \phi_{t}\right)=\operatorname{div}\left(T \operatorname{grad} \phi_{s}\right)+S
$$

The definitions of $\phi_{t}, \phi_{s}, T, S, a, b$ and the proper functional relationship of $\left(\phi_{t}-\phi_{s}\right)$ for different regions are given in table 4 .

\subsection{Simplifying assumptions}

The generalised mathematical statement (see $\$ 2.2$ to 2.8 ) is applicable to any melting or solidification problem. It is very.difficult to solve all these coupled equations together along with the generalised boundary conditions and so has not been tried so far. On the other hand, it is possible to simplify the generalised mathematical statement depending on the nature of the problem. In fact, all numerical studies so far are based on such assumptions which are directed to some specific applications. Broadly, there are four classes of simplifications possible and these are described in table 5. Based on these assumptions, the simplified mathematical formulations for different problems are listed in table 6.

One more important assumption is the steady state assumption. This assumption is useful for the physical understanding of many complex processes, specially rapid solidification processes like laser melting and solidification (Chan et al 1985; Basu \& Srinivasan 1988). Under steady state assumptions, the melting problem can be simplified by neglecting the latent heat of fusion and thus the solution can be obtained by sir. the interface. Such studies are mainly done to analyse the molten pool geometry and flow pattern in the pool.

\section{Methods of formulation}

\subsection{Variable domain methods}

As explained earlier, the energy conservation equation is derived with temperature as the dependent variable. The energy equation is solved in two regions ( $S$ and $L$ ) 


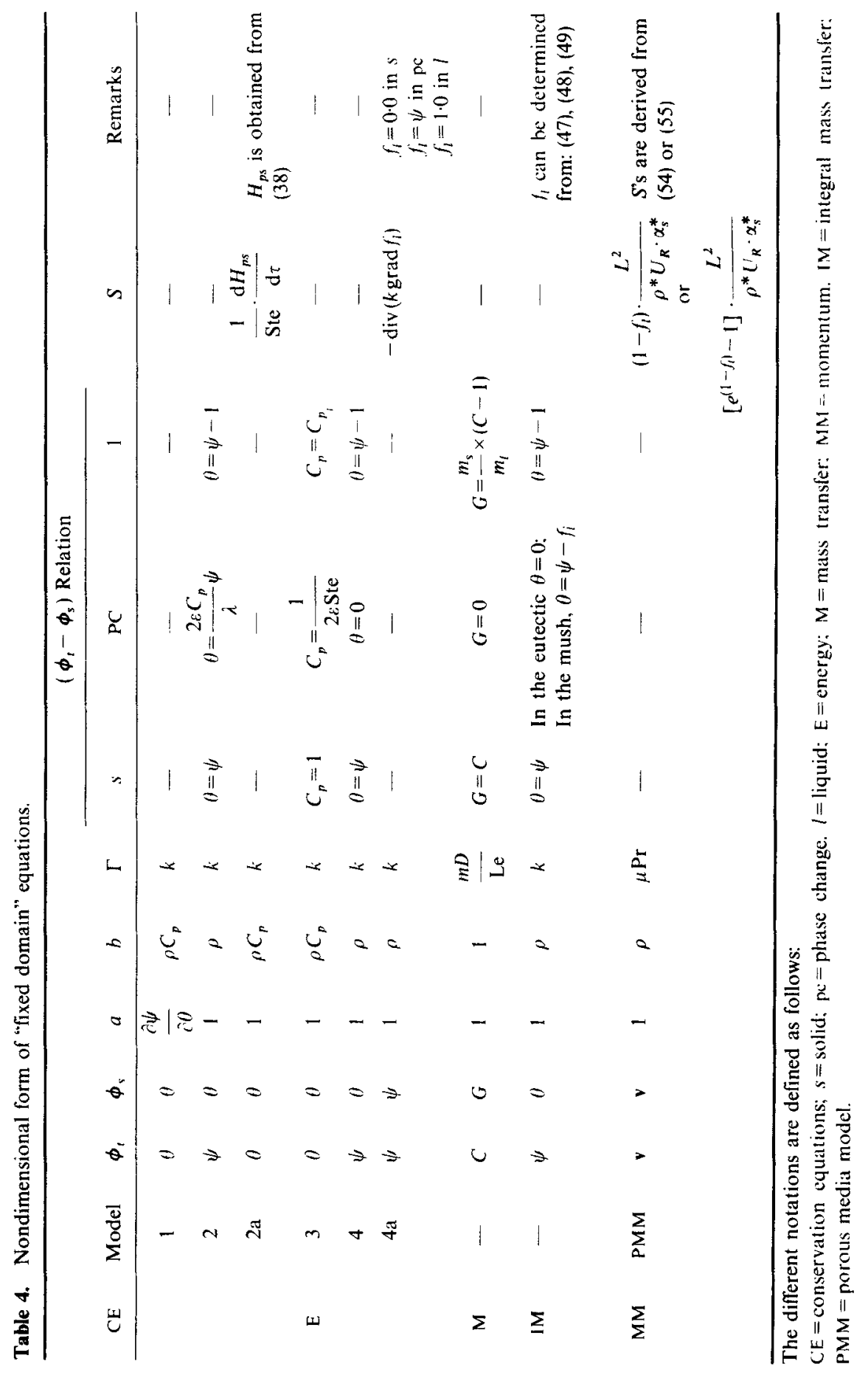


Table 5. Classification of simplifying assumptions.

\begin{tabular}{llrll}
\hline Notation & $\begin{array}{l}\text { Nature of } \\
\text { simplification }\end{array}$ & No. & Description & Remarks \\
\hline D & Dimensional & I & $\begin{array}{l}\text { One-dimensional } \\
\text { Two-dimensional }\end{array}$ & $\begin{array}{l}\text { Analytical solution possible } \\
\text { Most common assumption }\end{array}$ \\
P & Property & I & No property change & $\begin{array}{l}\text { Analytical solution possibte } \\
\text { in one dimension } \\
\text { No buoyancy force }\end{array}$ \\
G & II & $\begin{array}{l}\text { No density variation } \\
\text { All properties except density } \\
\text { are invariant }\end{array}$ & $\begin{array}{l}\text { Numerical solution is needed } \\
\text { Thermal diffusion controlled } \\
\text { Both thermal diffusion and } \\
\text { convection controlled }\end{array}$ & $\begin{array}{l}\text { Convection terms neglected } \\
\text { Mrocess }\end{array}$ \\
M & II & I & Pure metal & $\begin{array}{l}\text { Accurate solutions cannot be } \\
\text { obtained by fixed domain } \\
\text { formulation } \\
\text { Mass diffusion is important }\end{array}$ \\
\hline
\end{tabular}

separately and the solutions in the two regions are coupled through the energy balance at the interface. How to track or approximate the interface efficiently is the main question that is to be answered in all methods of the variable domain formulations.

There are five different groups depending on the ways of handling the interface. In the fixed grid method, a special differencing scheme is written near the interface considering the interface the boundary. Either grid sizes or the number of grids are adjusted in the variable space grid method so that the interface lies on a grid point or line. In the variable time step method the time step is selected such that the interface moves one grid per time-step. In the boundary immobilization method the interface remains fixed by transformation of co-ordinates. The isotherm migration method consists of exchanging one of the spatial co-ordinates with temperature, making the former a dependent variable and temperature the independent variable. All these methods will be discussed-only finite difference methods are considered.

3.1a Method of fixed grids: The usual way of solving the heat transfer equation over a fixed domain in one dimension by a finite difference method is to evaluate the temperature $\theta$ at the discrete grid points $(i \Delta x)$ on a fixed grid at different times $(\tau)$. The complication associated with a moving boundary is that at any time $(\tau)$ the moving boundary (e.g. the interface) is located between two neighbouring grid points, say, $i \Delta x$ and $(i+1) \Delta x$ (figure 7). In this method a special finite difference scheme based on unequal grid space interval is written near the interface. Let us assume that the interface is located at a distance $p x$ from the grid point i $\Delta x$ at a time, $\tau$. Using Lagrangian interpolation between the points $a_{0}, a_{1}$ and $a_{2}$ (figure 7), the space derivatives can be written as follows (Crank 1981).

For $x<I(\tau)$,

$$
\frac{\partial^{2} \theta}{\partial x^{2}}=\frac{2}{\Delta x^{2}}\left(\frac{\theta_{i-1}}{p+1}-\frac{\theta_{i}}{p}\right), \quad \text { at } x=i \Delta x,
$$




$$
\frac{\partial \theta}{\partial x}=\frac{1}{\Delta x}\left(\frac{p \theta_{i-1}}{p+1}-\frac{p+1}{p} \theta_{i}\right), \text { at } x=I(\tau) .
$$

[Both (56) and (57) are written with $\theta_{\text {interface }}=0$ (which follows from the definition of $\theta)$ ].

Similar expressions can be derived for $x>I(\tau)$. The interface energy balance equation will take the following form in one dimension,

$$
\left(k \frac{\partial \theta}{\partial x}\right)_{l}-\left(\frac{\partial \theta}{\partial x}\right)_{s}=-\frac{\operatorname{Re} \operatorname{Pr}}{\text { Ste }} \rho v_{I} .
$$

\begin{tabular}{|c|c|c|c|}
\hline Assumption & $\begin{array}{c}\text { Mathematical } \\
\text { statement }\end{array}$ & $\begin{array}{l}\text { Dependent } \\
\text { variables } \\
\& \text { operators }\end{array}$ & Remarks \\
\hline $\begin{array}{l}(\mathrm{DI}) \\
(\mathrm{PI}) \\
\text { (GI) } \\
\text { (MI) }\end{array}$ & $\begin{array}{c}\frac{\partial \phi}{\partial \tau}=\nabla^{2} \phi \\
\left.\nabla \phi\right|_{s} ^{l}=-\frac{\rho \cdot \operatorname{Re} \cdot \operatorname{Pr}}{\text { Ste }} \cdot \frac{\mathrm{d} I}{\mathrm{~d} \tau}\end{array}$ & $\begin{array}{c}\phi=\theta \\
\nabla^{2}=\frac{\partial^{2}}{\partial x^{2}}, \nabla=\frac{\partial}{\partial x}\end{array}$ & $\begin{array}{l}\text { Most simplified problem. } \\
\text { Analytical solution available } \\
\text { (Goodman 1958). Important } \\
\text { for casting problem }\end{array}$ \\
\hline $\begin{array}{l}\text { (DII) } \\
(\mathrm{PI}) \\
(\mathrm{GI}) \\
(\mathrm{MI})\end{array}$ & $\begin{array}{c}\frac{\partial \phi}{\partial \tau}=\nabla^{2} \phi \\
\left.\nabla \phi\right|_{s} ^{l}=-\frac{\rho \cdot \operatorname{Re} \cdot \operatorname{Pr} \mathrm{d} I}{\operatorname{Ste}} \cdot \frac{1}{\mathrm{~d} \tau}\end{array}$ & $\begin{array}{c}\phi=\theta \\
\nabla^{2}=\frac{\partial^{2}}{\partial x^{2}}+\frac{\partial^{2}}{\partial y^{2}} \\
\nabla=\frac{\partial}{\partial n}\end{array}$ & $\begin{array}{l}\text { Most common problem } \\
\text { attempted so far (Duda et } \\
\text { al 1975). Important for } \\
\text { casting, thermal storage }\end{array}$ \\
\hline $\begin{array}{l}\text { (DI) } \\
\text { (PII) } \\
\text { (GI) } \\
\text { (MI) }\end{array}$ & $\begin{array}{c}\frac{\partial \phi}{\partial \tau}=\nabla \cdot(\Gamma \nabla \phi) \\
\left.\nabla \phi\right|_{s} ^{l}=-\frac{\rho \cdot \operatorname{Re} \cdot \operatorname{Pr}}{\operatorname{Ste}} \cdot \frac{\mathrm{d} I}{\mathrm{~d} \tau}\end{array}$ & $\begin{array}{l}\phi=\theta \\
\Gamma=\alpha \\
\nabla=\frac{\partial}{\partial x}\end{array}$ & $\begin{array}{l}\text { This problem has been ana- } \\
\text { lysed by Goodrich (1978); } \\
\text { important for permafrost }\end{array}$ \\
\hline $\begin{array}{l}\text { (DII) } \\
\text { (PII) } \\
\text { (GI) } \\
\text { (MI) }\end{array}$ & $\begin{array}{c}\frac{\partial \phi}{\partial \tau}=\nabla \cdot(\Gamma \nabla \phi) \\
\left.\nabla \phi\right|_{s} ^{l}=-\frac{\rho \cdot \operatorname{Re} \cdot \operatorname{Pr}}{\operatorname{Ste}} \cdot \frac{\mathrm{d} I}{\mathrm{~d} \tau}\end{array}$ & $\begin{array}{c}\phi=\theta \\
\Gamma=\alpha \\
\nabla=i \frac{\partial}{\partial x}+j \frac{\partial}{\partial y}\end{array}$ & 一 \\
\hline $\begin{array}{l}\text { (DI) } \\
\text { (PIII) } \\
\text { (GI) } \\
\text { (MI) }\end{array}$ & $\begin{array}{c}\frac{\partial \phi}{\partial \tau}+\operatorname{Re} \cdot \operatorname{Pr} \nabla \cdot(u \phi)=\nabla \cdot(\Gamma \nabla \phi) \\
\left.\nabla \phi\right|_{s} ^{l}=-\frac{\rho \cdot \operatorname{Re} \cdot \operatorname{Pr}}{\operatorname{Ste}} \cdot \frac{\mathrm{d} I}{\mathrm{~d} \tau}\end{array}$ & $\begin{array}{l}\phi=\theta \\
\Gamma=\alpha \\
\nabla=\frac{\partial}{\partial x}\end{array}$ & $\begin{array}{l}\text { The velocity for volume } \\
\text { change can be determined } \\
\text { explicitly from interface } \\
\text { mass balance }\end{array}$ \\
\hline $\begin{array}{l}\text { (DII) } \\
\text { (PIII) } \\
\text { (GI) } \\
\text { (MI) }\end{array}$ & $\begin{array}{c}\frac{\partial \phi}{\partial \tau}+\operatorname{Re} \cdot \operatorname{Pr} \nabla \cdot(u \phi)= \\
\nabla \cdot(\Gamma \nabla \phi)+S \\
\left.\nabla \phi\right|_{s} ^{l}=-\frac{\rho \cdot \operatorname{Re} \cdot \operatorname{Pr} \mathrm{d} I}{\operatorname{Ste}} \cdot \frac{\bar{d} \tau}{\mathrm{d} \tau}\end{array}$ & $\begin{array}{c}\phi=\theta, u, v \\
\Gamma=\alpha, v \\
\nabla=i \frac{\partial}{\partial x}+j \frac{\partial}{\partial y}\end{array}$ & $\begin{array}{l}\text { One has to solve momen- } \\
\text { tum equations to determine } \\
\text { velocity distribution due to } \\
\text { volume change; so proper } \\
\text { assumption is (DII) (PIII) } \\
\text { (CII) (MI) }\end{array}$ \\
\hline
\end{tabular}

Table 6. Simplified mathematical formulation. 
Table 6. (Continued)

\begin{tabular}{|c|c|c|c|}
\hline Assumption & $\begin{array}{l}\text { Mathematical } \\
\text { Statement }\end{array}$ & $\begin{array}{l}\text { Dependent } \\
\text { variables } \\
\& \text { operators }\end{array}$ & Remarks \\
\hline $\begin{array}{l}\text { (DII) } \\
\text { (PIII) } \\
\text { (GII) } \\
\text { (MI) }\end{array}$ & 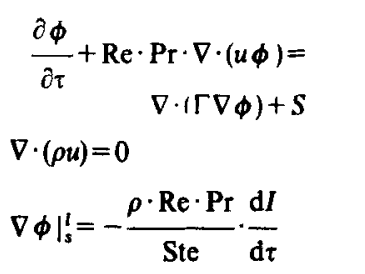 & $\begin{array}{l}\Gamma=\alpha, v \\
\nabla=i \frac{\partial}{\partial x}+j \frac{\partial}{\partial y}\end{array}$ & $\begin{array}{l}\text { This is the most practical } \\
\text { problem suitable for many } \\
\text { important applications: cast- } \\
\text { ing, welding, crystal growth } \\
\text { (Oreper \& Szekely 1984; } \\
\text { Kuo \& Sun 1985) }\end{array}$ \\
\hline $\begin{array}{l}\text { (DI) } \\
\text { (PI) } \\
\text { (GI) } \\
\text { (MII) }\end{array}$ & $\begin{aligned} \frac{\partial \phi}{\partial \tau} & =\nabla^{2} \phi \\
\left.\nabla \phi\right|_{s} ^{l} & =-\frac{\rho \cdot \operatorname{Re} \cdot \operatorname{Pr}}{\operatorname{Ste}} \cdot \frac{\mathrm{d} I}{\mathrm{~d} \tau} \\
\left.\nabla \phi\right|_{s} ^{l} & =-\frac{\operatorname{Re} \cdot \operatorname{Pr}}{\mathrm{Le}} \cdot \frac{\mathrm{d} I}{\mathrm{~d} \tau}\end{aligned}$ & $\begin{aligned} \phi & =\theta, C \\
\nabla^{2} & =\frac{\partial^{2}}{\partial x^{2}} \\
\nabla & =\frac{\partial}{\partial x}\end{aligned}$ & $\begin{array}{l}\text { For this problem also an } \\
\text { analytical solution is possi- } \\
\text { ble (Tien \& Geiger 1967) }\end{array}$ \\
\hline $\begin{array}{l}\text { (DII) } \\
\text { (PII) } \\
\text { (GI) } \\
\text { (MII) }\end{array}$ & $\begin{array}{c}\frac{\partial \phi}{\partial \tau}=\nabla \cdot(\Gamma \nabla \phi) \\
\left.\nabla \phi\right|_{s} ^{l}=-\frac{\rho \cdot \operatorname{Re} \cdot \operatorname{Pr}}{\operatorname{Ste}} \cdot \frac{\mathrm{d} I}{\mathrm{~d} \tau} \\
\left.\nabla \phi\right|_{s} ^{l}=-\frac{\operatorname{Re} \cdot \operatorname{Pr}}{\mathrm{Le}} \cdot \frac{\mathrm{d} I}{\mathrm{~d} \tau}\end{array}$ & $\begin{array}{l}\phi=\theta, C \\
\Gamma=\alpha, D \\
\nabla=i \frac{\partial}{\partial x}+j \frac{\partial}{\partial y}\end{array}$ & $\begin{array}{l}\text { This is important for the } \\
\text { alloy solidification problem } \\
\text { where convection is suppress- } \\
\text { ed for fine microstructure } \\
\text { (Reddy \& Sekhar 1985) }\end{array}$ \\
\hline $\begin{array}{l}\text { (DII) } \\
\text { (PIII) } \\
\text { (GII) } \\
\text { (MII) }\end{array}$ & $\begin{array}{l}\frac{\partial \phi}{\partial \tau}+\nabla \cdot(u \phi) \cdot \operatorname{Re} \operatorname{Pr}= \\
\nabla \cdot(\Gamma \nabla \phi)+S \\
\nabla \cdot(\rho u)=0 \\
\left.\nabla \phi\right|_{s} ^{l}=-\frac{\rho \cdot \operatorname{Re} \cdot \operatorname{Pr}}{\operatorname{Ste}} \cdot \frac{\mathrm{d} I}{\mathrm{~d} \tau} \\
\left.\nabla \phi\right|_{s} ^{l}=-\frac{\operatorname{Re} \cdot \operatorname{Pr}}{\mathrm{Le}} \cdot \frac{\mathrm{d} I}{\mathrm{~d} \tau}\end{array}$ & $\begin{array}{l}\phi=\theta, C, u, v \\
\Gamma=\alpha, D, v \\
\nabla=i \frac{\partial}{\partial x}+j \frac{\partial}{\partial y}\end{array}$ & $\begin{array}{l}\text { This is the most generalised } \\
\text { problem (Beckermann \& } \\
\text { Viskanta 1988) }\end{array}$ \\
\hline
\end{tabular}

For multidimensional problems, Patel (1968) has derived a generalised interface condition and for a two-dimensional problem, the conditions are as follows;

$$
\begin{aligned}
& {\left[1+\left(\frac{\partial I}{\partial x}\right)^{2} \cdot\right]\left[\left(k \frac{\partial \theta}{\partial y}\right)_{l}-\left(\frac{\partial \theta}{\partial y}\right)_{s}\right]=-\frac{\operatorname{Re} \operatorname{Pr}}{\text { Ste }} \rho v_{I y},} \\
& {\left[1+\left(\frac{\partial I}{\partial y}\right)^{2}\right]\left[\left(k \frac{\partial \theta}{\partial x}\right)_{l}-\left(\frac{\partial \theta}{\partial x}\right)_{s}\right]=-\frac{\operatorname{RePr}}{\text { Ste }} \rho v_{l x} .}
\end{aligned}
$$

Lazardis (1970) used this method in multidimensional problems where he assumed a quadratic temperature profile near the interface to avoid the singularity of the finite difference equations when $p=0$. Lazardis determined the coefficients of the quadratic profile using the following two conditions, 


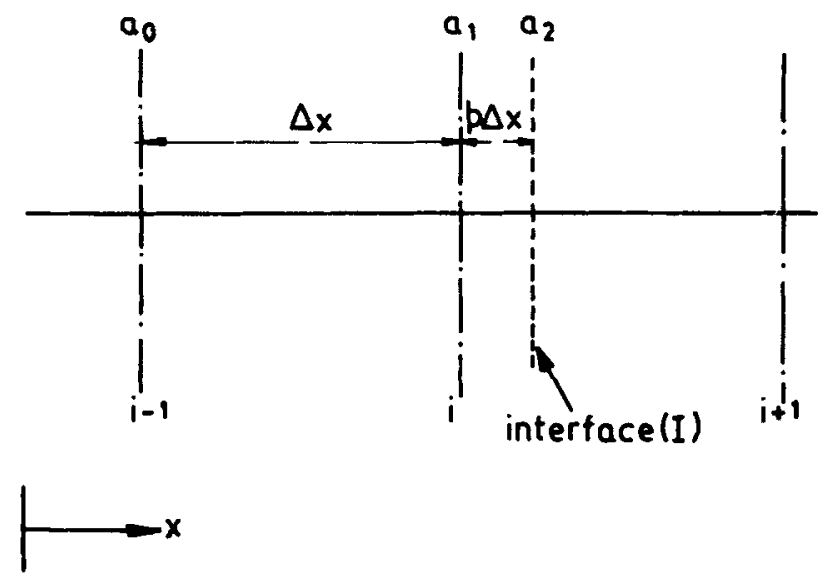

Figure 7. Grid arrangements for the method of fixed grids.

a) the time rate of change of the temperature vanishes at the interface

$$
\left(\left.\frac{\partial \theta}{\partial \tau}\right|_{t}=0 ;\right)
$$

b) the temperature gradient along the interface is zero $\left(\left.\because \theta\right|_{I}=0\right)$.

The two-dimensional problem chosen was that of an infinitely long prism with fixed temperature and convective cooling boundary conditions (figure 8). Koh et al (1969) used this method while analysing the thermal responses of space vehicle walls during re-entry. Using a one-dimensional problem, they analysed this problem where two interfaces (e.g. solid/liquid and liquid/vapour) exist.

While solving a problem of frost penetration into earth (figure 9) by the fixed grid method, Goodrich (1978) suggested a new solution procedure based on nodal iteration which reduces the computation time. Except the interface and its

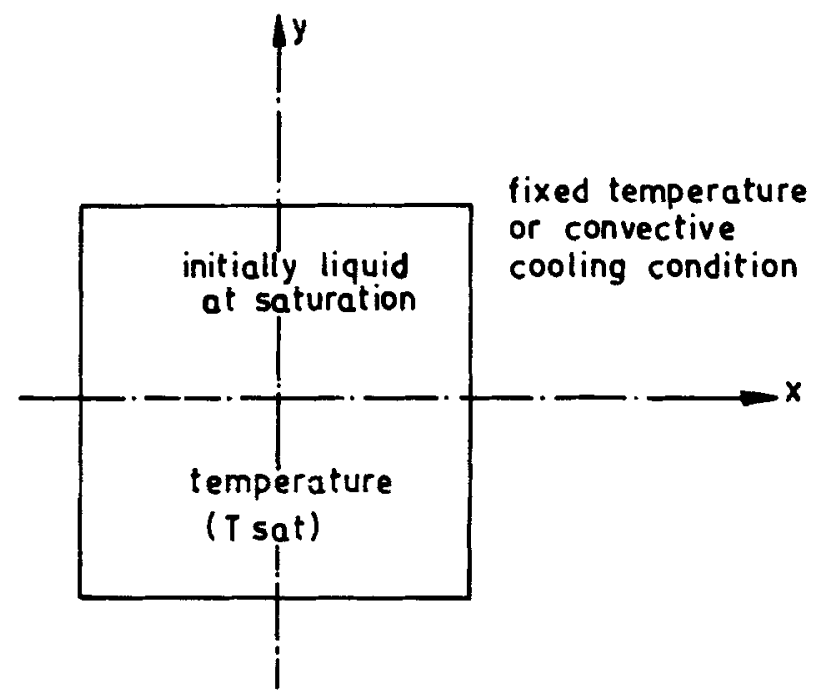

Figure 8. Lazardis's (1970) and Gupta \& Kumar`s (1985) physical problem. 

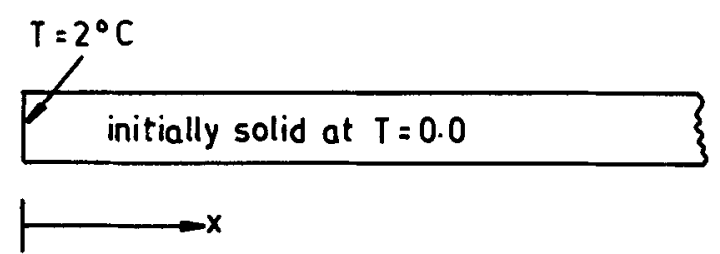

property values $K s=2.25 \mathrm{w} / \mathrm{mc}$

$$
\begin{aligned}
C_{P_{5}} & =1.5 \mathrm{MJ} / \mathrm{m}^{3} \mathrm{c} \\
C_{P_{1}} & =2.5 \mathrm{MJ} / \mathrm{m}^{3} \mathrm{c} \\
K I & =1.75 \mathrm{~W} / \mathrm{mc} \\
\lambda & =100 \mathrm{MJ} / \mathrm{m}^{3}
\end{aligned}
$$

Figure 9. Goodrich's (1978) physical problem.

neighbours, solution of other grid points were obtained by either a forward or backward Gauss elimination method. The solution of the interface and its neighbours was found by an iterative method which he called "nodal iteration". Rao \& Sastri (1983) also used Goodrich's (1978) solution procedure to solve a different one-dimensional problem. Later, Rao \& Sastri (1984) extended Goodrich's (1978) method to two dimensions while solving a solidification problem in an infinitely long square prism with the fluid initially at superheated conditions.

3.1b Methods of variable space grids: In this method, the number of space intervals are kept constant and the space intervals are adjusted in such a manner that the interface lies on a particular grid point. The space interval is thus a function of time. Differentiating temperature partially with respect to time following a given grid line instead of at constant $x$, one obtains,

$$
\frac{\partial \theta}{\partial \tau}=\left.\frac{\partial \theta}{\partial x}\right|_{i} \frac{\partial x}{\partial \tau}+\left.\frac{\partial \theta}{\partial \tau}\right|_{x} .
$$

A general grid point $\left(x_{i}\right)$ moves according to the following relation,

$$
\frac{\mathrm{d} x_{i}}{\mathrm{~d} \tau}=\frac{x_{i}}{I(\tau)} \cdot \frac{\mathrm{d} I}{\mathrm{~d} \tau}
$$

Hence, for one-dimensional problems, the governing equations will be modified as follows,

$$
\left.\frac{\partial \theta}{\partial \tau}\right|_{i}=-\frac{x_{i}}{I(\tau)} \cdot \frac{\mathrm{d} I}{\mathrm{~d} \tau} \cdot \frac{\partial \theta}{\partial x}+\frac{\partial^{2} \theta}{\partial x^{2}}
$$

Murray \& Landis (1954) used this formulation to solve the problem of freezing by the explicit method. Heitz \& Westwater (1970) also used this method to solve a onedimensional problem of solidification with the liquid initially at saturated temperature. They incorporated the volume change (e.g. shrinkage during solidification) and a higher value of liquid thermal conductivity to simulate the effect of fluid flow. Though multidimensional problems are more complex, one can obtain a very good insight into these from their work. Using this method in two 
dimensions, Springer \& Olson (1962), Rathjen \& Jiji (1971) and Tien \& Wilkes (1970) have obtained solutions of several two-dimensional problems. This method has also been used by many researchers for finite element analysis. The papers of Bannerot \& Jamet $(1975,1977)$ and Jamet (1978) are of this type.

3.1c Method of variable time grid: In this method, the time-step is calculated in such a manner that the interface moves one grid spacing per time-step. This is described now with a one-dimensional problem. The problem is physically defined in figure 10. It can be mathematically described as,

$$
\partial \theta / \partial \tau=\partial^{2} \theta / \partial x^{2} ; \quad 0 \geqslant x \geqslant I(\tau)
$$

and the corresponding boundary conditions are,

$$
\left.\begin{array}{ll}
-\partial \theta / \partial x=Q \theta+R & ; x=0, \tau>0 \\
\theta=0 & ; I(\tau)<x \leqslant 1 \cdot 0, \tau>0 \\
\rho \operatorname{Re} \operatorname{Pr}(\partial x / \partial \tau)=\text { Ste } \cdot(\partial \theta / \partial x) & ; x=I(\tau), \tau>0 \\
I(0)=0 . &
\end{array}\right\}
$$

Integrating (64) over $x$ from 0 to $I$ and using the boundary conditions, the following integral equation results

$$
Q \int_{0}^{\tau} \theta \mathrm{d} \tau+R \tau=\frac{\operatorname{Re} \operatorname{Pr} \rho I(\tau)}{\text { Ste }}-\int_{0}^{I(\tau)} \theta \mathrm{d} x
$$

With an initial guess of $\Delta \tau^{(0)}$, the finite difference form of (64) can be solved with the corresponding boundary and initial conditions. If the interface position is $(i \Delta x)$ at the old time-level then the interface should be at $(i+1) \Delta x$ after the current timelevel. Substituting $I(\tau)$ by $(i+1) \Delta x$ along with the new temperature solution, one can obtain a new time step, $\Delta \tau^{(1)}$, from (66). If $\Delta \tau^{(0)}$ and $\Delta \tau^{(1)}$ match, then $\Delta \tau^{(1)}$ is the required time-step. Otherwise, the iteration is continued till $\Delta \tau^{k}$ and $\Delta \tau^{k+1}$ match to certain accuracy.

Douglas \& Gallie (1970) used this method to solve a one-dimensional problem with a simplified boundary condition as

$$
\partial \theta / \partial x=-1 ; \quad x=0, \tau>0 .
$$

Gupta \& Kumar (1981) used the generalised boundary conditions [e.g., (65)] to solve a one-dimensional problem. Goodling \& Khader (1974) used the interface balance condition to find the required time-step. According to them, the required time-step is as follows:

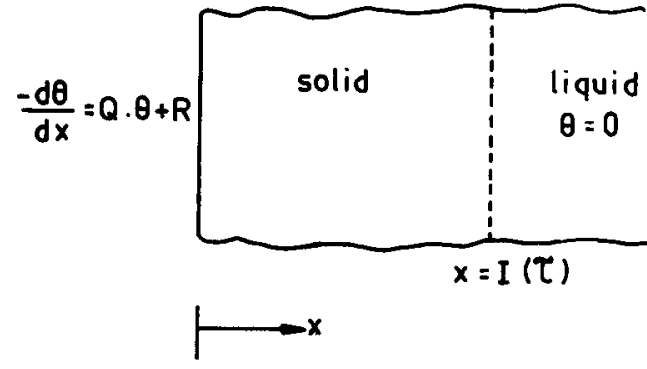

Figure 10. A physical model for a solidification problem with liquid initially at saturated state 


$$
\Delta \tau^{(1)}=[\rho \operatorname{Re} \operatorname{Pr}(i+1) \Delta x] /\left(\left.\operatorname{Ste} \frac{\partial \theta}{\partial x}\right|_{I}\right)
$$

They checked the convergence through the boundary condition (65). Gupta \& Kumar (1981) followed the same method as Goodling \& Khader (1974) but used (68) for checking the convergence while the $\Delta \tau$ was obtained from (66). Both Goodling \& Khader's (1974) and Gupta \& Kumar's (1981) methods require a starting solution. Gupta \& Kumar (1981), however, suggested a way of calculating the initial guess value of $\Delta \tau$. On comparison of all these methods, Douglas \& Gallie's (1974) and Gupta \& Kumar's (1981) methods are found to be superior to others. This is because of the fact that the required time-step has been checked through the integral form of the energy equation while the other methods are based on boundary conditions only.

Golder \& Gay (1975) used this method while analysing the batch melting process in a glass manufacturing industry. Later, Voller \& Cross (1981) used this method in enthalpy formulation.

3.1d Boundary immobilization method: This is the most powerful method in variable domain formulation. Frequently finite difference methods are more straightforward in application to the problems governed by nonlinear partial differential equations in a fixed region of fixed extent than they are to linear equations in a changing domain. Using this fact, the space variable is changed in the boundary immobilization method in order to fix the moving interface. Under the transformation, the governing equations which are linear become nonlinear partial differential equations.

This method will now be explained with a one-dimensional problem of icemelting shown in figure 11. Using the transformation $\xi=x / I(\tau)$, the governing equation takes the following form:

$$
I^{2}(\partial \theta / \partial \tau)-\xi I(\mathrm{~d} I / \mathrm{d} \tau) \cdot(\partial \theta / \partial \xi)=\left(\partial^{2} \theta / \partial \xi^{2}\right) .
$$

The second term on the left hand side of (69) requires further explanation. We note

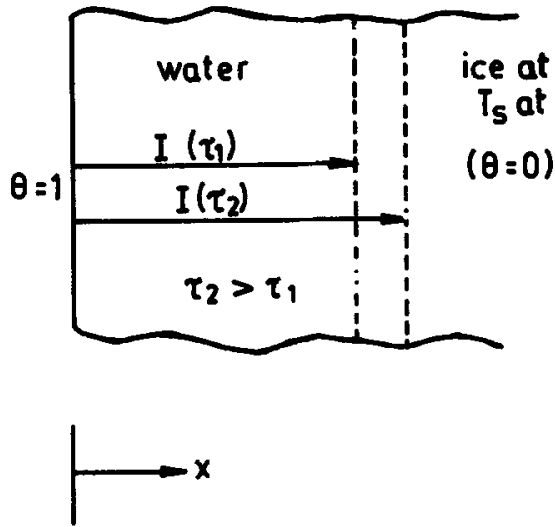

(a)

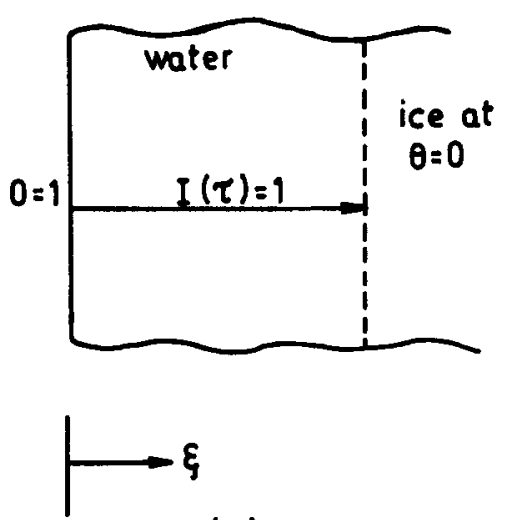

(b)

Figure 11. A physical model for a melting problem in (a) physical and (b) transformed planes. 
that this term is similar to the convectic term. The control volume (or the grid points) are stationary in the transformed plane $(\xi, \tau)$. But the control volumes are moving in the physical plane $(X, \tau)$ and this results in convective flux in the transformed governing equation. This term can be called the "pseudo-convection" term. In a multidimensional problem, there will be some more cross-derivatives in the final differential equation other than the pseudo-convection terms. We will show these terms through the equation $(\lambda, \xi)$ of Duda et al (1975) transformed from the equation in the $(r-z)$ co-ordinate which is as follows (see figure 12),

$$
\begin{aligned}
\frac{\partial \theta}{\partial \tau}= & \frac{\partial^{2} \theta}{\partial \lambda^{2}}+\frac{1}{\lambda} \cdot \frac{\partial \theta}{\partial \lambda}-\frac{2 \xi}{H_{t}} \cdot \frac{\partial H_{t}}{\partial \lambda} \frac{\partial^{2} \theta}{\partial \xi \partial \lambda}+\frac{\partial^{2} \theta}{\partial \xi^{2}}\left[\frac{1}{H_{t}^{2}}+\frac{\xi^{2}}{H_{t}^{2}} \cdot\left(\frac{\partial H_{t}}{\partial \lambda}\right)^{2}\right]+ \\
& +\frac{\partial \theta}{\partial \xi}\left[\frac{2 \xi}{H_{t}} \cdot\left(\frac{\partial H}{\partial \lambda}\right)^{2}-\frac{\xi}{H_{t}} \cdot \frac{\partial^{2} H_{t}}{\partial \lambda^{2}}+\frac{\xi}{H_{t}} \cdot \frac{\partial H_{t}}{\partial \tau}\right] .
\end{aligned}
$$

For understanding the different terms, (70) can be rearranged as follows,

$$
\begin{aligned}
\frac{\partial \theta}{\partial \lambda}= & \frac{\partial^{2} \theta}{\partial \lambda^{2}}+\frac{1}{\lambda} \cdot \frac{\partial \theta}{\partial \lambda}+\frac{1}{H_{t}^{2}} \cdot \frac{\partial^{2} \theta}{\partial \xi^{2}}+[\underbrace{\left[\frac{\xi}{H_{t}} \cdot \frac{\partial H_{t}}{\partial \tau} \cdot \frac{\partial \theta}{\partial \xi}\right]}_{\mathrm{A}}+ \\
& +\underbrace{\left[\frac{\partial H}{\partial \lambda}\left(\frac{\xi^{2}}{H_{t}^{2}} \cdot \frac{\partial H_{t}}{\partial \lambda} \frac{\partial^{2} \theta}{\partial \xi^{2}}-\frac{2 \xi}{H_{t}} \cdot \frac{\partial^{2} \theta}{\partial \xi \cdot \partial \lambda}+\frac{2 \xi}{H^{2}} \frac{\partial H_{t}}{\partial \lambda} \frac{\partial \theta}{\partial \xi}\right)+\frac{\xi}{H} \frac{\partial^{2} H_{t}}{\partial \lambda^{2}}\right]}_{\mathrm{B}} .
\end{aligned}
$$

We note that the term " $\mathrm{A}$ " is the "pseudo-convection" term. The term "B" is due to the non-orthogonality of the control volumes in the transformed plane $(\lambda, \xi)$ with respect to the physical plane $(r, z)$. According to $\mathrm{Hsu}$ et al $(1981)$, these can be designated as pseudo-anisotropic diffusion terms.

Referring again to our one-dimensional formulation [e.g. (69)], the advantage of transformation is now obvious. The moving boundary has become a fixed one (figure 11). The resulting finite difference equations along with the initial and boundary conditions can be solved by any standard finite difference technique where $\partial I / \partial \tau$ is obtained from the interface boundary condition.

Saitoh (1978) presented an immobilization method similar to that of Duda et al (1975). But in Saitoh's formulation the domain boundary shape can also be selected arbitrarily, thereby allowing the application of the method to diverse physical problems. While solving two-dimensional solidification problems in $(r-\theta)$ coordinates, Saitoh (1978) used the following transformation (figure 13),

$$
\eta=[\mathrm{r}-I(\theta, \tau)] /[B(\theta)-I(\theta, \tau)] .
$$

Thus an arbitrary region is mapped into a rectangle, regardless of $\theta$, with $\eta=0$ at the interface and $\eta=1$ at the fixed boundary. He solved the freezing problem in square, triangular and elliptic cavities under different cooling rates.

Hsu et al (1981) suggested a control volume-based formulation. Identifying the pseudo-convection and pseudo-anistropic diffusion terms, they formulated the 


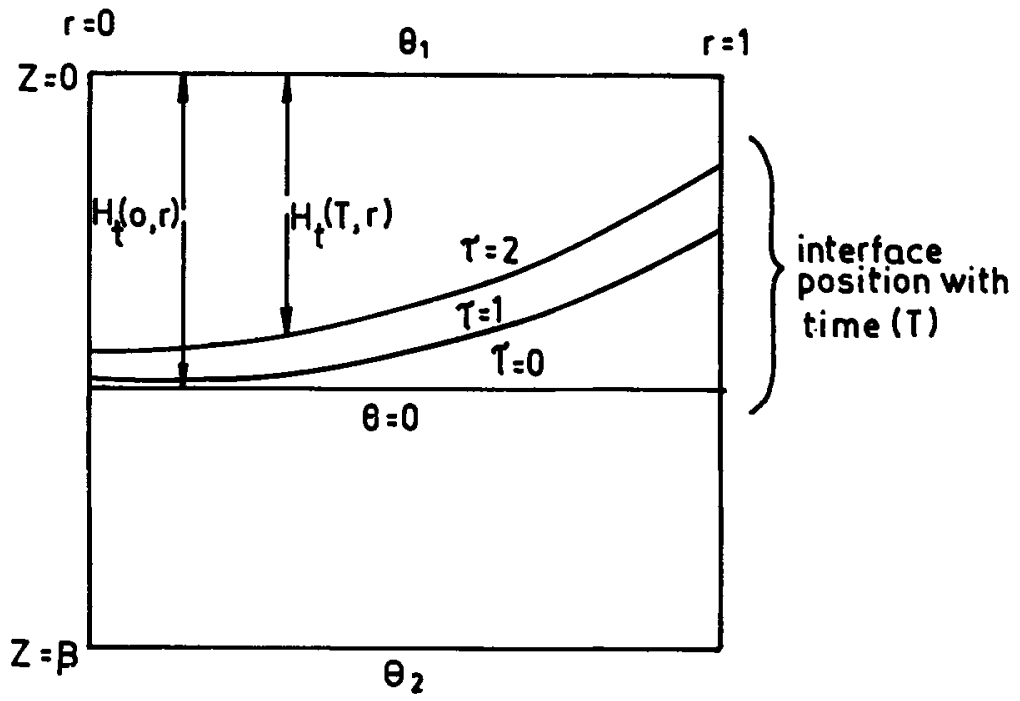

(a)

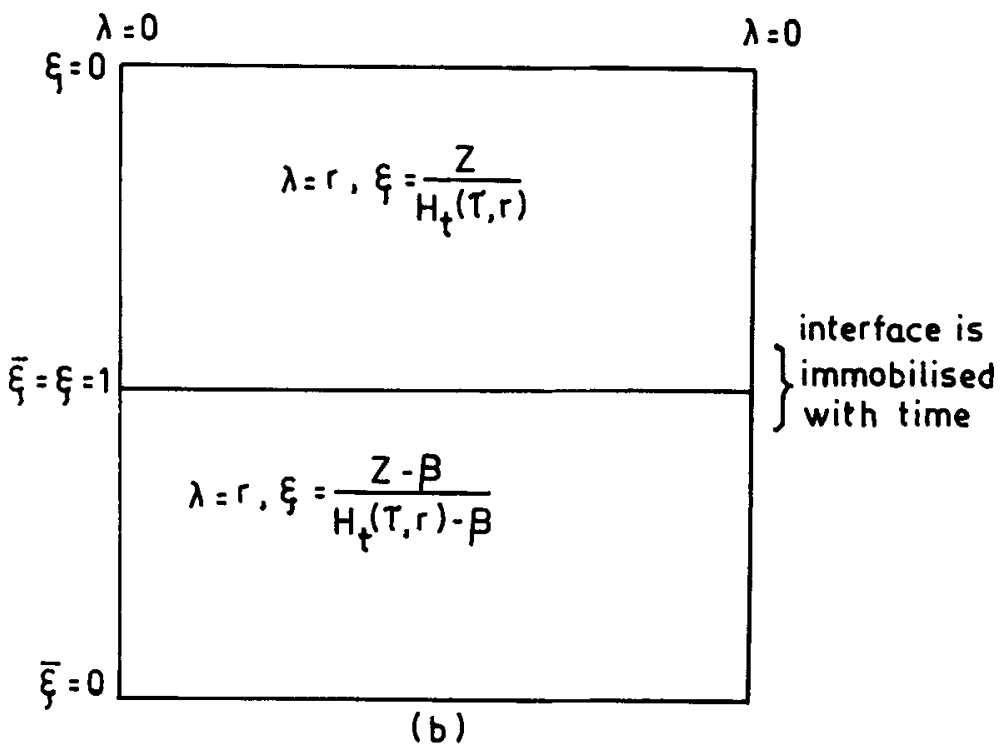

Figure 12. Duda el al's (1975) problem in (a) physical and (b) transformed planes.

equations in a cylindrical co-ordinate system and treated different terms separately. They included the effect of convection through a convective heat transfer coefficient at the interface and the modified interface balance equation is as follows,

$$
\left(k^{*} \frac{\partial T^{*}}{\partial n^{*}}\right)_{l}-\left(k^{*} \cdot \frac{\partial T^{*}}{\partial n^{*}}\right)_{s}=-\rho^{*} \lambda \cdot v_{I}^{*}+h_{I}^{*}\left(T_{b l}^{*}-T_{m}^{*}\right)
$$

In a subsequent paper, Sparrow \& Hsu (1981) implemented this formulation to solve a thermal storage problem. The advantage of Hsu et al's (1981) formulation is 


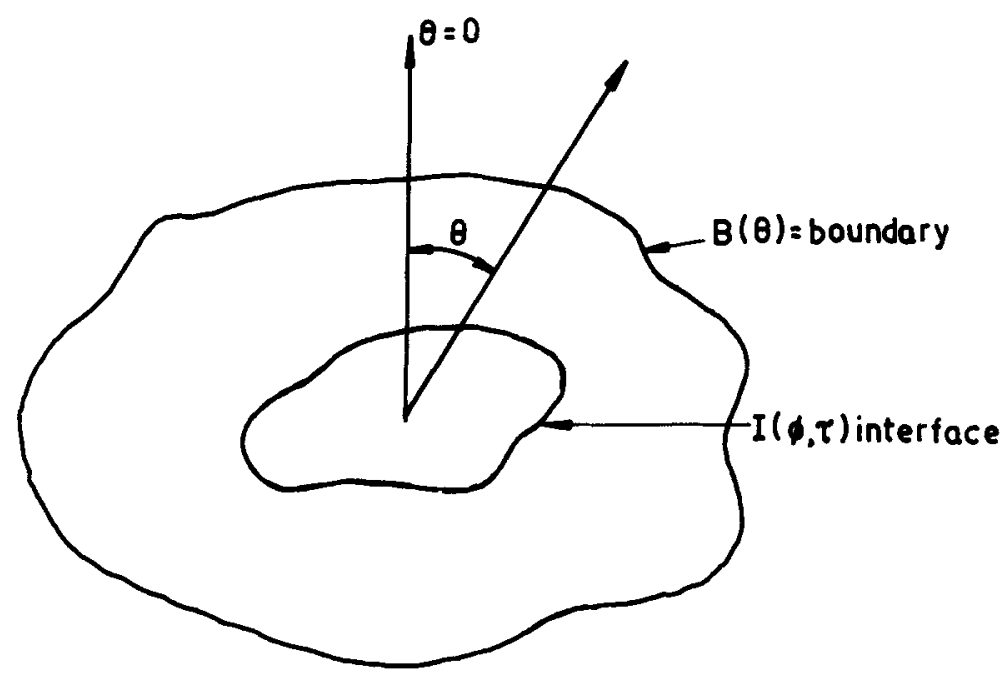

Figure 13. Saitoh's (1978) model in a physical plant.

that the final equation reflects the physical processes governing the melting/solidification problems.

Gupta \& Kumar (1985) used this method while analysing solidification in an infinitely long square prism initially filled with liquid at saturated temperature. The problem in both physical and transformed planes along with the transformation is shown in figure 14. Szekely \& Chabbra (1970) used this method in a onedimensional problem introducing the heat transfer coefficient at the interface to include the effect of natural convection. Using the standard natural convection correlation, they obtained very good agreement between experimental and

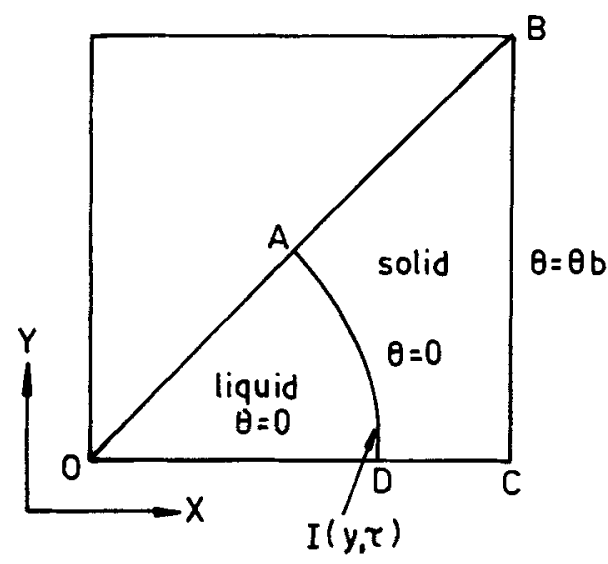

(a)

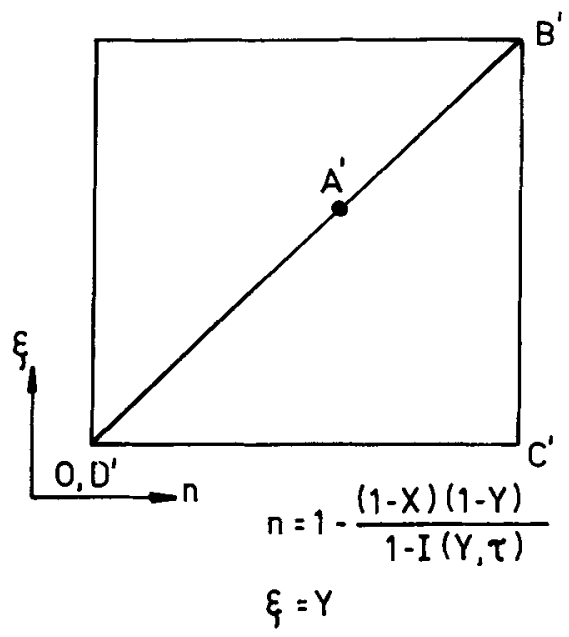

(b)

Figure 14. Gupta \& Kumar's (1985) model in (a) physical and (b) transformed planes. 
numerical results. Sparrow \& Souza Mendis (1982) and Chiesa \& Guthrie (1974) used experimentally determined correlations for heat transfer coefficients. The correlations are as follows.

For energy storage problems (Sparrow \& Souza Mendis 1982),

$$
\mathrm{Nu}_{x}=0.486 \mathrm{Ra}_{x}^{0.25}\left[\left(T_{\infty}^{*}-T_{m}^{*}\right) /\left(T_{0}^{*}-T_{m}^{*}\right)\right]^{1.25},
$$

and Chiesa \& Guthrie's (1974) correlation,

$$
\mathrm{Nu} / \mathrm{Nu}_{0}=\exp \left[-7 \cdot 8\left(L R_{f r} / \alpha \mathrm{Nu}\right)\right]
$$

where $\mathrm{Nu}_{0}=$ Nusselt number for steady state systems $=0.078(0.68)^{\mathrm{L} / \mathrm{D}} \mathrm{Ra}^{1 / 3}$

For further details regarding diffusion controlled melting and solidification problems see Sproster (1981) and Spaid et al (1971).

Sparrow et al (1977) analysed the effect of natural convection during melting around a cylinder. They neglected the cross-terms in the final equation to reduce the computational complexity. Energy and momentum (in primitive variable) equations were solved in the melt by the semi-implicit method (see $\S 4 \cdot 1 \mathrm{c}$ ). Through extensive numerical experiments over a wide range of parameters (e.g., $7 \times 10^{4} \leqslant \operatorname{Ra} \leqslant 7 \times 10^{6}, 7 \leqslant \operatorname{Pr} \leqslant 70$ and $0.05 \leqslant$ Ste $\leqslant 0.15$ ), they concluded that $\operatorname{Pr}$ and Ste do not have any significant influence on wall heat flux. But Ra strongly modifies the wall heat flux and the interface shape. Their analysis clearly shows the effect of convection during melting.

Ramchandran et al (1981) studied solidification in a rectangular enclosure in the presence of natural convection. Assuming a quasi-stationary process, they used vorticity-transport formulation to solve momentum equations and studied the effect of Rayleigh number $\left(10^{3} \leqslant \mathrm{Ra} \leqslant 10^{5}\right)$ and Biot number $(0 \cdot 5 \leqslant \mathrm{Bi} \leqslant 2 \cdot 0)$. Later, Ramchandran et al (1982) solved the same problem with top and bottom surface insulated. Gadgil \& Gopin (1984) analysed a melting problem similar to that of Sparrow et al (1977) but for a higher Rayleigh number range $\left(10^{6} \leqslant \mathrm{Ra} \leqslant 10^{8}\right)$. Rieger et al (1982) analysed melting around a cylinder in the presence of natural convection by the numerical grid generation method. The predicted trends are consistent with the observation, but no direct comparison of predicted and measured results is given.

Kroeger \& Ostrach (1974) analysed a continuous casting process by conformal mapping. They used vorticity-transport formulation to solve momentum equations and obtained results for $\mathrm{Gr}_{h}=10^{4}$ to $10^{6}, \mathrm{Re}=50.0, \mathrm{Pr}=0.02$ and $\mathrm{Pe}$ (based on withdrawal speed $)=0 \cdot 5,1 \cdot 0$. It was found that there is negligible effect of convection on the interface profile though the flow pattern changes dramatically between $\mathrm{Gr}=10^{4}$ and $10^{6}$.

Benard et al (1985) studied the effect of convection during melting in a rectangular enclosure. They used the model developed by Gadgil \& Gobin (1984) and validated the model by comparing experimental and numerical results. Recently, Benard et al (1986) modified Gadgil \& Gobin's (1984) model by retaining the cross-terms in the solid phase governing equation. This is because of the strong influence of the interface shape on the isotherm pattern in the solid phase. Two important conditions for applying the quasi-stationary assumption were suggested as follows:

$$
\left.\begin{array}{l}
\left(\operatorname{Ra}^{0.25} \text { Ste } \rho_{l}^{*}\right) / \rho_{s}^{*} \geqslant 1.0 \\
\left(0.33 \text { Ste } \rho_{l}^{*}\right) / \rho_{s}^{*} \leqslant 1.0
\end{array}\right\}
$$


These two conditions are very useful because most of the analysis of melting and solidification with convection are based on the "quasi-stationary" assumption.

3.1e Isotherm migration method: In this method, the heat flow equation is written in a form which concentrates attention on the movement of isotherms. Temperature is exchanged with one of the space variables making temperature an independent variable. This method is analogous to the Lagrangian formulation of fluid flow phenomenon. In one dimension, the governing equation takes the form,

$$
\partial x / \partial \tau=(\partial x / \partial \theta)^{-2}\left(\partial^{2} x / \partial \theta^{2}\right)
$$

This equation along with any boundary condition can be solved by any standard finite difference method. The interface position will be obtained from the value of $x$ corresponding to $\theta=0$. The transformed governing equation is more complicated for multidimensional problems and for a two-dimensional problem the governing equation is

$$
(\partial y / \partial \tau)=-\left[\left(\partial^{2} \theta / \partial x^{2}\right)-\left(\partial^{2} y / \partial \theta^{2}\right)(\partial y / \partial \theta)^{-3}\right](\partial y / \partial \theta)
$$

Crank \& Gupta (1975) used this method to solve a two-dimensional solidification problem of a saturated liquid in an infinitely long square prism. They started their solution by a one-parameter integral method of Poots (1962). They used $y$ as a dependent variable which has to be a single-valued function. For convection dominated problems, this criterion is likely to fail and thereby restricts the use of this method to such problems. Crank \& Crowley (1978) suggested a novel way of implementing the isotherm migration method. Using the fact that heat flow will always be normal to the isotherms, they wrote the governing equation in terms of a local co-ordinate treating the isotherm element as part of a cylindrical system. By geometry, they related the local co-ordinate to the global co-ordinate assuming the isotherm element to be a straight line. They used the local radial co-ordinate as a dependent variable. This method requires a starting solution and they solved Lazardis's (1970) problem using Crowley's (1978) model for the starting solution. Later, Crank \& Crowley (1979) suggested an implicit formulation based on the Crank-Nicholson scheme. While comparing the implicit and explicit methods, they found that the computation time for the implicit method was larger than that of the explicit scheme when the number of grids on a particular isotherm is more than 10 . This is really an unusual phenomenon which they also could not explain conclusively.

\subsection{Fixed domain methods}

The formulation of fixed domain methods has already been discussed in $\S 2.7$. In brief, energy conservation is written in terms of enthalpy and diffusion terms are evaluated by temperature in fixed domain methods so that a single energy equation is valid in all the zones including the interface. Besides the physical explanation, the fixed domain concept can also be explained mathematically. Enthalpy is continuous only in time whereas temperature is continuous only in space. Hence, the enthalpy formulation, where the time derivative is evaluated in enthalpy and the space derivative is calculated in temperature, is valid in all regions.

Dusinberre (1945) and Eyres et al (1946) were the first to report the application of the enthalpy formulation for melting and solidification. Price \& Slack (1954) used 
enthalpy formulation for freezing in a semi-infinite plane. Albasiny (1956) solved a solidification problem in a finite slab and Baxter (1962) used this method for simulating solidification of cylinders.

Szekely \& Themlis (1970) proposed the model 1 while Atthey (1974) used the model 2a (see $\S 2.7 \mathrm{a}$ ). Meyer (1971) proposed model 2 and demonstrated the strength of this model by solving a two-dimensional melting problem for two values of $\varepsilon$, e.g., 0.5 and $10^{-6}$. The heating and cooling curves for these two values of $\varepsilon$ were matched and therefore Meyer (1971) concluded that this method is independent of $\varepsilon$. However, Voller et al (1979) showed that model 2a is dependent on the chosen value of $\varepsilon$. While solving Goodrich's (1978) problem by model 2 , they found oscillations in the transient temperature profiles. Crowley (1978) solved Lazardis's (1970) problem by this model. Comini et al (1974) and Morgan et al (1978) applied model 2 using the finite element method.

While solving Goodrich's (1978) problem by the model 2, Voller \& Cross (1981a) observed a stepwise increase in temperature. They argued that the way in which the two-phase control volumes were handled was not proper. Shamsundar (1978) also observed waviness in the heat flux predictions while solving a one-dimensional problem with this model. This behaviour is characteristic of the enthalpy model and it is caused by holding the temperature of a two-phase node/control volume constant at $T_{m}^{*}$. By keeping the temperature constant at $T_{m}^{*}$ for a two-phase node/control volume, the heat flux calculation at that node/control volume is not correct and, as a result, waviness in the temperature and heat flux histories occur. Voller \& Cross (1981a) modified the solution scheme of the enthalpy equation to eliminate this waviness in the solution. They determined the time-step at each timelevel in such a way that the interface moves from one node/control volume to another. They called this scheme the "node jumping scheme" and this way of solving enthalpy equations can be broadly called the "variable time-step enthalpy formulation". They solved Lazardis's (1970) problem to test the solution scheme. For materials which melt over a range of temperatures, they used model $2 \mathrm{a}$ and solved a spot-welding problem. In a subsequent paper, Voller \& Cross (1981b) solved a two-dimensional solidification problem in a cylindrical co-ordinate system using model 2. Through a numerical experiment, they reported a simple way of calculating total solidification time for two-dimensional geometry. Bell (1982) calculated local solidification time of a control volume by an analytical method and showed that the time over which temperature, obtained by enthalpy formulation, remains constant is equal to the solidification time of that control volume. He has thus justified Voller \& Cross's (1981a) argument about the stepwise increase of temperature. Voller (1985) used the model $2 \mathrm{a}$ to propose an efficient implicit finite difference scheme for enthalpy formulation which incorporates the node jumping procedure. He solved a one-dimensional solidification problem with liquid initially at superheated condition.

Shamsundar \& Sparrow (1975a) proposed model 4 (table 4) and showed the equivalence between enthalpy and temperature solution. They solved a twodimensional solidification problem with saturated liquid for thermal storage application. They studied the effect of Ste and $\mathrm{Bi}$ on the interface movement. Later, Shamsundar \& Sparrow (1975b) included the effect of density change in the enthalpy formulation and analysed a casting problem to study the cavity formation. Hsu et al $(1978,1980)$ and Kou et al (1981) used model 4 to study various surface heating problems with stationary or moving heat source. Recently, Ramarao \& 
Sekhar (1987) solved the problem of surface solidification with a moving heat source by this model. Basu \& Date (1987) proposed model 4a (see table 4) and solved a one-dimensional problem. They showed that for the same problem, model $4 a$ with a direct solution technique is more efficient than model 4 .

Bonacina et al (1975) used model 3 to solve a one-dimensional solidification problem with liquid initially in a saturated and superheated state. They used $\varepsilon=$ 0.25 and observed that the results are insensitive to $\varepsilon$ when liquid is initially in the saturated state. Comini et al (1974) used this model in finite element analysis while Morgan et al (1978) modified Comini et al's (1974) formulation to eliminate certain numerical problems. Rolphe \& Bathe (1982) used model $2 \mathrm{~b}$ in finite element analysis.

Application of enthalpy formulation for the convection dominated problem has been first tried by Morgan (1981) who used the finite element method. Identifying the liquid elements, he solved the momentum equations only in the liquid element with proper boundary conditions. This is obviously the most common approach and Voller et al (1987) called this approach the "switch off" technique. Gartling (1980), who also used the finite element method, used the variable viscosity method for solving momentum equations. The main problem in this method is the proper $\mu^{*}\left(T^{*}\right)$ function in the phase change region because $\mu^{*}\left(T^{*}\right)$ in the phase change region is implicitly dependent on the interface position. Voller et al (1987) have also mentioned this difficulty in assigning proper $\mu^{*}\left(T^{*}\right)$ in the phase change region. Kou \& Sun (1985) used the variable viscosity method to solve a melting problem in two dimensions by the finite difference method. They studied the effect of different forces, e.g., electromagnetic, buoyancy and surface tension forces, on the weld pool shape. Later Kou \& Wang (1986a and b) extended Kou \& Sun's (1985) formulation to three dimensions and solved some typical welding problems. Voller et al (1987) compared the different methodologies for solving momentum equations along with the enthalpy formulation. For alloys, they implemented a porous media model and have shown the validity of the porous media model by comparing results obtained by other methods. They used linear and nonlinear forms of Darcy's law but further research is needed to determine the proper Darcy's law which, in turn, is dependent on the geometry of the mush.

Oreper \& Szekely (1984) used the "switch off" technique for momentum equations with model 3 of enthalpy formulation to study heat and fluid flow during welding. Using steel as the material, they assumed a linear increase of specific heat in the phase change region and solved the momentum equations by vorticity-stream function formulation. They have studied the effect of electromagnetic, buoyancy and surface tension forces on the total heat transfer and pool shape. Oreper et al (1986) used this formulation to study the transient growth and collapse of axisymmetric weld pools in spot-welding operations. From the transient growth rate and heat flux at the interface, they predicted the order of the secondary dendrite arm spacings.

\subsection{Alloy solidification problems}

Different ways of alloy solidification formulation are already described in $\S 2.7$. The concept of using a potential function for evaluating diffusion terms was first proposed by Fix (1978) through a variable $G(=C / m)$ which is continuous across the 
mushy region. He suggested this formulation but did not, however, test it through some standard problem. Crowley \& Ockendon (1979) formulated the alloy solidification problem following Fix's approach and termed the variable $G$ the chemical activity. They determined the temperature in the mushy region assuming that temperature and enthalpy are related through partitioned values. They also assumed the phase diagram to be linear. A one-dimensional problem was solved and they compared their results with the analytical one which is based on the similarity solution. Meyer (1981) improved Crowley \& Ockendon's (1979) model by including an actual phase diagram (e.g., nonlinear $C^{*}-T^{*}$ relationship) and presented a one-dimensional model. Recently, Voller (1987) implemented a nodejumping scheme in Crowley \& Ockendon's (1979) formulation. With the nodejumping scheme, Voller (1987) could eliminate the oscillations in the concentration profiles observed in Crowley \& Ockendon's (1979) result. But Voller's (1987) scheme is only applicable for plane front movement which is rarely true.

Grange et al (1976) used a mass balance approach while solving a onedimensional solidification of the water-ice system. Sekhar et al (1983) solved a twodimensional alloy solidification problem by this method. The amount of eutectic was found from the phase diagram. They used a linearised Scheil equation, i.e.

$$
f_{l}=\left(T_{m}^{*}-T^{*}\right) /\left(T_{m}^{*}-T_{l}^{*}\right)
$$

This assumption is not valid for alloys of low solute content (e.g. less than $10 \%$ for the Al-Cu system, Flemings 1974). Recently, Basu \& Sekhar (1988) modified Sekhar et al's (1983) formulation by solving the nonlinear Scheil equation. They validated their formulation by simulating an experimental study; Reddy \& Sekhar (1985). They have carried out a wide range of numerical experiments to study the effect of Biot number and initial solute concentration on the total solidification time.

For further references on one-dimensional models, the papers of Huppert \& Worster (1985), Worster (1986) and Hunt \& McCartney (1987) are important. But all these models are difficult to extend beyond specially formulated one-dimensional studies.

\subsection{Summary}

All methods based on variable domain formulation predict the interface profile accurately whereas interpolation is required to find the interface in the fixed domain method. Multidimensional problems can be efficiently handled by the fixed domain method without much difficulty. Formulation of multidimensional problems by variable domain methods are complex. With the boundary immobilisation method, there are some difficulties in the formulation of the multidimensional problem in a finite domain. Though no analysis considered the effect of curved interface, variable domain methods, mainly boundary immobilisation, are preferable because of accurate estimation of interface profile.

For alloy solidification problems, the variable domain methods are very difficult to use because of the existence of four zones, namely, solid, eutectic, mush and liquid. Since there are two moving interfaces-solidus and liquidus, it will be very difficult to implement boundary immobilization to fix the position of both the interfaces. On the other hand, a fixed domain method can be easily applied because of well-defined enthalpy-temperature relationships in the four zones during an alloy 
solidification problem. As a result, all the existing alloy solidification models are based on fixed domain formulations.

The significant drawback of fixed domain formulation is the stepwise increase of temperature, enthalpy etc. with time when pure metal is used for analysis. As discussed earlier, this problem arises because of keeping the temperature of the phase change control volume constant (e.g. at saturation temperature) till the phase change control volume releases or absorbs all the latent heat. For control volumes of finer size, this assumption is close to physical reality and thus the fixed domain formulation predicts correct temperature and enthalpy histories with fine grids. For coarse grid solution, this assumption leads to improper calculation of heat fluxes and results in a stepwise increase of temperature, enthalpy etc. Hence, fixed domain formulation is dependent on the size of the space-grids or one has to incorporate variable time-step approach in the enthalpy formulation. Unfortunately both these alternatives would increase the computation time. Further research to eliminate this drawback of fixed domain methods for pure metal solution is needed. For alloys, the fixed domain method always predicts correct temperature and enthalpy histories because alloy melts or solidifies over a range of temperatures.

For solving momentum equations in the liquid region during melting and solidification, a perfect and generalised method is yet to be developed. Boundary immobilization is the most accurate one because of precise calculation of the interface geometry. As mentioned earlier, this method is difficult to implement for multidimensional problems with finite domain and also for alloy solidification problems. On the other hand, all the techniques based on enthalpy formulation are approximate. In particular, the porous media model which is based on the proper Darcy equation, requires further research. For example, information about the geometry of the mushy region (e.g., the size and shape of dendrites) is essential in order to use the proper Darcy equation during alloy solidification. One has to develop a perfect mushy region model first and then couple this model with the momentum equation to calculate the flow in the mushy region.

\section{Methods of solution}

In this section, finite difference equations for different formulations and subsequently the solution procedure will be described. Stability and convergence criteria of different schemes are also highlighted.

\subsection{Variable domain method}

4.1a Explicit scheme: The explicit finite difference equations of the different formulations for any one-dimensional problem can be represented by the following generalised form,

$$
\phi_{i}^{n+1}=A_{i} \phi_{i}^{n}+B_{i} \phi_{i+1}^{n}+C_{i} \phi_{i-1}^{n} .
$$

The definitions of $\phi$ and expressions of $A_{i}, B_{i}$ and $C_{i}$ for different formulations are given in table 7 .

The significant drawback of the explicit difference scheme is the restriction on the time-step for stability. The stability criterion at any interior node is (Roach 1976),

$$
\Delta \tau \leqslant \Delta^{2} / G
$$


Table 7. Coefficients of explicit finite difference equations for the "variable domain" formulation.

\begin{tabular}{lccll}
\hline Method & $\phi$ & \multicolumn{1}{c}{$A_{i}$} & \multicolumn{1}{c}{$B_{i}$} & \multicolumn{1}{c}{$C_{i}$} \\
\hline $\begin{array}{l}\text { Fixed grids \& variable } \theta \\
\text { time-grids }\end{array}$ & $1-\left(2 \Delta \tau / \Delta x^{2}\right)$ & $\left(\Delta \tau / \Delta x^{2}\right)$ & $\left(\Delta \tau / \Delta x^{2}\right)$ \\
Variable space grids & $\theta$ & $1-2\left(\Delta \tau / \Delta x^{2}\right)$ & $\left(\Delta \tau / \Delta x^{2}\right)+M \cdot(\Delta \tau / \Delta x)$ & $\left(\Delta \tau / \Delta x^{2}\right)-M \cdot(\Delta \tau / \Delta x)$ \\
$\begin{array}{l}\text { Boundary immobili- } \\
\text { zation }\end{array}$ & $\theta$ & $1-\left(2 \Delta \tau / I^{2} \cdot \Delta \xi^{2}\right)$ & $\left(\Delta \tau / I^{2} \Delta \xi^{2}\right)+(N \cdot \Delta \tau / \Delta \xi)$ & $\left(\Delta \tau / I^{2} \cdot \Delta \xi^{2}\right)-(N \cdot \Delta \tau / \Delta \xi)$ \\
Isotherm migration & $x$ & $1-\left(2 P \cdot \Delta \tau / \Delta \theta^{2}\right)$ & $\left(P \cdot \Delta \tau / \Delta \theta^{2}\right)$ & $\left(P \cdot \Delta \tau / \Delta \theta^{2}\right)$ \\
\hline
\end{tabular}

The different notations are defined as follows:

$M=x_{i} \cdot\left(I^{n}-I^{n-1}\right) /\left(I^{n} \cdot \Delta \tau\right) ; \quad N=\xi_{i} \cdot\left(I^{n}-I^{n-1}\right) /\left(I^{n} \cdot \Delta \tau\right) ; \quad P=\left[\Delta \theta^{2} /\left(x_{i+1}^{n}-2 x_{i}^{n}+x_{i-1}^{n}\right)\right]^{2}$.

where $\Delta$ is the spatial step size and $G=2,4,8$ for one-, two- and three-dimensional problems. The stability criterion is of the same form as (81) corresponding to the finite difference equation based on the boundary condition but $G$ may be smaller than that specified above (Shamsundar 1978).

For the variable space grid and boundary immobilisation methods, an additional stability criterion has to be satisfied because of pseudo convection terms. Comparing the governing equations of the variable space grid (63) and boundary immobilisation (69) methods with that of a one-dimensional convection-diffusion equation, the additional stability criterion is,

$$
B \Delta x / C<2 \text {, }
$$

where, $B=[(i-1) /(I-1)] \cdot(\mathrm{d} I / \mathrm{d} \tau)$ for the variable space grid,

$=[(i-1) / I] \cdot \Delta \xi$ for boundary immobilisation,

$C=1$ for the variable space grid,

$=1 / I^{2}$ for boundary immobilisation.

4.1b Implicit scheme: The implicit finite difference equations for all these methods can be expressed as

$$
A_{i} \phi_{i+1}^{n+1}+B_{i} \phi_{i}^{n+1}+C_{i} \phi_{i-1}^{n+1}=D_{i} .
$$

$\phi$ is $x$ in case of the isotherm migration method and $\theta$ for all other methods. The coefficients $A_{i}, B_{i}, C_{i}$ and $D_{i}$ for different methods are given in table 8. When (83) is applied to all the grid points, a set of algebraic equations result. The solution procedure of the algebraic equations will be described later. The implicit finite difference equation is unconditionally stable.

4.1c Semi-implicit scheme: In addition to fully explicit and implicit methods, there is a semi-implicit method referred to as the quasi-stationary approach in the last section. In this approach, the location of $I(T)$ is predicted implicitly over some interval and the governing equations are then solved implicitly on the predicted domain. Huber (1939) first used this approach, hence it is sometimes called "Huber's method". While comparing fully implicit and semi-implicit methods, 
Table 8. Coefficients of implicit finite difference equations for "variable domain" formulations.

\begin{tabular}{llclc}
\hline Method & \multicolumn{1}{c}{$A_{i}$} & $B_{i}$ & $C_{i}$ & $D_{i}$ \\
\hline $\begin{array}{l}\text { Fixed grid \& va- } \\
\text { riable time-grid }\end{array}$ & $-\Delta \tau / \Delta x^{2}$ & $1+\left(2 \Delta \tau / \Delta x^{2}\right)$ & $-\Delta \tau / \Delta x^{2}$ & $\theta_{i}^{n}$ \\
Variable space grids & $-\left[\left(\Delta \tau / \Delta x^{2}\right)+M \cdot(\Delta \tau / \Delta x)\right]$ & $1+\left(2 \Delta \tau / \Delta x^{2}\right)$ & $-\left[\left(\Delta \tau / \Delta x^{2}\right)-M \cdot(\Delta \tau / \Delta x)\right]$ & $\theta_{i}^{n}$ \\
$\begin{array}{l}\text { Boundary immobi- } \\
\text { lization }\end{array}$ & $-\left[\left(\Delta \tau / I^{2} \Delta \xi^{2}\right)+(N \Delta \tau / \Delta \xi)\right]$ & $1+\left(2 \Delta \tau / I^{2} \Delta \xi^{2}\right)$ & $-\left[\left(\Delta \tau / I^{2} \Delta \xi^{2}\right)-(N \Delta \tau / \Delta \xi)\right]$ & $\theta_{i}^{n}$ \\
$\begin{array}{l}\text { Isotherm migra- } \\
\text { tion }\end{array}$ & $-P \Delta \tau / \Delta \theta^{2}$ & $1+\left(2 P \Delta \tau / \Delta \theta^{2}\right)$ & $-P \Delta \tau / \Delta \theta^{2}$ & $x_{i}^{n}$ \\
\hline
\end{tabular}

The different notations are defined as follows:

$M=x_{i}\left(I^{n+1}-I^{n}\right) /\left(I^{n+1} \cdot \Delta \tau\right) ; \quad N=\xi_{i}\left(I^{n+1}-I^{n}\right) /\left(I^{n+1} \cdot \Delta \tau\right): P=\left[\Delta \theta^{2} /\left(x_{i+1}^{n+1}-2 x_{i}^{n+1}+x_{i-1}^{n+1}\right)\right]^{2}$.

Meyer (1978) has shown that the semi-implicit method would break down when the interface moves rapidly. Meyer (1978) considered a problem where the liquid is $100^{\circ} \mathrm{C}$ above the saturation temperature and solved this problem by both implicit and semi-implicit methods with two different latent heats (one 200 times greater than the other). He found that the solution, using the semi-implicit method, with low latent heat (e.g., faster interface movement) is totally unstable whereas the high latent heat solution is stable. This shows that the application of the semi-implicit method is problem-dependent and the condition for applying this method has been reported by Benard et al (1986) which is mentioned in the last section.

\subsection{Fixed domain method}

4.2a Explicit scheme: The explicit finite difference equations in one dimension for all the models are expressed as follows,

$$
\phi_{t, i}^{n+1}=\phi_{t, i}^{n}+P\left[\phi_{s, i+1}^{n}-2 \phi_{s, i}^{n}+\phi_{s, i-1}^{n}\right]+S_{i}^{n} .
$$

The definitions of $\phi_{t}, \phi_{s}$ and the expressions of $P, S_{i}^{n}$ for different models are given in table 9.

All the finite difference equations are written with no property variations. The interface condition in all these models is built in the form of the $\psi-\theta$ relation in models 1,2 and $4, S_{i}^{n}$ in models $2 \mathrm{a}$ and $4 \mathrm{a}$ and $C_{p}-\theta$ in model 3 . The nodal latent

Table 9. Coefficients of explicit finite difference equations for the "fixed domain" formulation.

\begin{tabular}{lcclc}
\hline Model & $\phi_{t}$ & $\phi_{s}$ & \multicolumn{1}{c}{$P_{i}$} & $S_{i}$ \\
\hline 1 & $\theta$ & $\theta$ & $\left(\Delta \tau / \Delta x^{2}\right) \cdot[1 /(\mathrm{d} \psi / \mathrm{d} \theta)]$ & - \\
2 & $\psi$ & $\theta$ & $\Delta \tau / \Delta x^{2}$ & - \\
$2 \mathrm{a}$ & $\theta$ & $\theta$ & $\Delta \tau / \Delta x^{2}$ & $(1 / \mathrm{Ste}) \cdot\left(H_{p, s}^{n}-H_{p s}^{n-1}\right)$ \\
3 & $\theta$ & $\theta$ & $\left(\Delta \tau / \Delta x^{2}\right) \cdot\left(1 / C_{p}\right)$ & - \\
4 & $\psi$ & $\theta$ & $\Delta \tau / \Delta x^{2}$ & - \\
$4 \mathrm{a}$ & $\psi$ & $\psi$ & $\Delta \tau / \Delta x^{2}$ & $\left(\Delta \tau / \Delta x^{2}\right)\left(f_{l, i+1}^{n}-2 f_{l, i}^{n}+f_{l, i-1}^{n}\right)$
\end{tabular}


heat content, $H_{p s}$, is evaluated as follows,

$$
\left.\begin{array}{l}
H_{p s}=0.0 ; \quad \theta<0.0 \\
H_{p s}=1.0 ; \quad \theta>0.0
\end{array}\right\} .
$$

In the phase change control volumes (Voller et al 1979),

$$
H_{p s}^{n+1}=H_{p s}^{n}+\left(\theta_{i}^{n+1}-\theta_{i}^{n}\right)
$$

where

$$
\theta_{i}^{n+1}=2 \varepsilon\left(H_{p s}^{n}-0 \cdot 5\right)
$$

The stability criterion of (84) is the same as in $\$ 4.1 \mathrm{a}$.

4.2b Implicit scheme: Except for models 2 and 4, implicit finite difference equations for all other models can be written as follows,

$$
A_{i} \phi_{i+1}^{n+1}+B_{i} \phi_{i}^{n+1}+C_{i} \phi_{i-1}^{n+1}=D_{i} \text {. }
$$

The definitions of $\phi$ and expressions of $A_{i}, B_{i}, C_{i}$ and $D_{i}$ for all the models are shown in the table 10 . Since proper $(\psi-\theta)$ relations have to be maintained during calculation, the implicit finite difference equation for the models 2 and 4 changes from grid to grid depending on the state of the respective grid.

The detailed description of the solution methodology for pure metal and alloy has been reported by Shamsundar \& Sparrow (1975a) and Basu \& Sekhar (1988) respectively.

\section{2c Node-jumping scheme}

In the node-jumping scheme, the time-step is determined in such a manner that the interface jumps from one node to the next one during a time-step. If at a time $\tau$, the interface is at a node $p$, the latent heat content of the control volumes are,

$$
H_{p s}^{*}\left(H_{p s}\right)=\left\{\begin{array}{l}
0 \cdot 0(0 \cdot 0), \text { if } i=\text { solid } \\
\lambda / 2(0 \cdot 5), \text { if } i=p \\
\lambda(1 \cdot 0), \text { if } i=\text { liquid }
\end{array}\right\}
$$

Hence, the time-step selected should be such that the interface moves from $p$ to $p+1$ and the nodal latent heats are,

Table 10. Coefficients of implicit finite equations of the "fixed domain" formulations.

\begin{tabular}{lrllll}
\hline Model & $\phi$ & \multicolumn{1}{c}{$A_{i}$} & \multicolumn{1}{c}{$B_{i}$} & \multicolumn{1}{c}{$C_{i}$} & \multicolumn{1}{c}{$D_{i}$} \\
\hline 1 & $\theta$ & $-\left(\Delta \tau / \Delta x^{2}\right) \cdot[1 /(\mathrm{d} \psi / \mathrm{d} \theta)]$ & $1+\left\{2 \Delta \tau / \Delta x^{2} \cdot[(\mathrm{d} \psi / \mathrm{d} \theta)]\right\}$ & $-\left(\Delta \tau / \Delta x^{2}\right) \cdot[1 /(\mathrm{d} \psi / \mathrm{d} \theta)]$ & $\theta_{i}^{n}$ \\
$2 \mathrm{a}$ & $\theta$ & $-\left(\Delta \tau / \Delta x^{2}\right)$ & $1+\left(2 \Delta \tau / \Delta x^{2}\right)$ & $-\left(\Delta \tau / \Delta x^{2}\right)$ & $\theta_{i}^{n}-\left(H_{p s}^{n+1}-H_{p s}^{n}\right)$ \\
3 & $\theta$ & $-\left(\Delta \tau / \Delta x^{2}\right) \cdot\left(1 / C_{p}\right)$ & $1+\left[2 \Delta \tau /\left(\Delta x^{2} \cdot C_{p}\right)\right]$ & $-\left(\Delta \tau / \Delta x^{2}\right) \cdot\left(1 / C_{p}\right)$ & $\theta_{i}^{n}$ \\
$4 \mathrm{a}$ & $\psi$ & $-\left(\Delta \tau / \Delta x^{2}\right)$ & $1+2 \Delta \tau / \Delta x^{2}$ & $-\Delta \tau / \Delta x^{2}$ & $\psi_{i}^{n}-\left(\Delta \tau / \Delta x^{2}\right) \times$ \\
& & & & $\times\left(f_{l, i+1}^{n+1}-2 f_{l, i}^{n+1}+\right.$ \\
& & & & $\left.+f_{l, i-1}^{n+1}\right)$ \\
\hline
\end{tabular}




$$
H_{p s}^{*}\left(H_{p s}\right)=\left\{\begin{array}{l}
0 \cdot 0(0 \cdot 0), \text { if } i=\text { solid } \\
\lambda / 2(0 \cdot 5), \text { if } i=p+1 \\
\lambda(1 \cdot 0), \text { if } i=\text { liquid }
\end{array}\right\} .
$$

Therefore, an amount of $\lambda / 2$ has to be evolved from the control volumes $p$ and $p+1$. The source term $S_{i}^{n}$ of $(84)$ for the model 2 will take the following form,

$$
S_{i}^{n}=\left\{\begin{array}{l}
0 \cdot 0, i \neq p, i \neq p+1, \\
0 \cdot 5, \text { otherwise }
\end{array}\right.
$$

Starting from a guessed time-step, the correct time-step is found by an iterative procedure using the following relation to find the subsequent time-step (Voller et al 1979),

$$
\Delta \tau_{k}=\Delta \tau_{k-1}-\left[\theta_{p}^{n+1}\left(\Delta \tau_{k-1}-\Delta \tau_{k-2}\right) /\left(\theta_{p_{k-1}}^{n+1}-\theta_{p_{k-2}}^{n+1}\right)\right] .
$$

Here $\theta_{p}^{n+1}$ is calculated from (86). The iterative procedure is continued until the value of $\Delta \tau$ is such that

$$
\theta_{p}^{n+1}<\gamma,
$$

where $\gamma$ is the convergence factor.

The node jumping scheme can also be used in model 4 (Voller \& Cross 1981a), where the subsequent time-step is obtained from the following equation,

$$
\Delta \tau_{k}^{n+1}=\Delta \tau_{k-1}^{n+1}+\omega\left[\Delta \tau_{c}-\Delta \tau_{k-1}^{n+1}\right]
$$

where

$$
\begin{aligned}
\Delta \tau_{c} & =2 \psi_{p}^{n+1} \Delta \tau_{k-1}^{n+1}, \\
k & =\text { iteration level. }
\end{aligned}
$$

The correct time will be such that $\psi_{p}^{n+1}=0.5$ when the interface is at node $p$.

\subsection{Solution procedure}

The finite difference equations resulting from implicit formulation can be solved in two ways,

(i) point by point solution; and

(ii) line by line solution.

The line by line solution procedure can also be divided into three groups depending on the method of sweeps and these are as follows,

(i) single line sweep;

(ii) multiple line sweep; and

(iii) diagonal sweep.

The point by point iteration procedure is known as the Gauss-Seidal iterative method and for further details one can refer to Patankar (1980). For onedimensional problems, a line by line solution gives a direct solution and hence it is sometimes known as "direct solution" method. The Thomas algorithm (Roach 1976), is used in the line by line solution procedure. The algorithm requires the coefficient matrix to be tri-diagonal and for one dimensional problems the coefficient matrix is always tri-diagonal. But in two-dimensional problems the coefficient 
matrix is block tri-diagonal. This matrix is not easy to solve and the popular method of attack is iterative. Although the use of implicit methods allows a larger time-step, it generally takes many iterations to solve for that step. There is thus nothing to gain by just using the implicit method without an efficient solution algorithm. In two- and three-dimensional problems, one may use the alternative direction implicit (ADI) scheme instead of a fully implicit scheme. The ADI scheme makes use of the splitting of the time-step to obtain a multidimensional implicit method which requires the inversion of the tri-diagonal matrix.

The Thomas algorithm with a single-line sweep is the most common way of solving implicit finite difference equations. But multiple lines and diagonal sweeps are sometimes found to be useful in reducing the number of iterations. Godbole \& Date (1985) presented a comparative study between multiple line (two and three), diagonal, ADI and Gauss-Seidal iterations while solving a two-dimensional single phase diffusion problem with different boundary conditions. They have found that the Thomas algorithm with a two-line sweep is always efficient because of faster convergence. In melting and solidification problems, multiple line and diagonal sweeps have not been tried so far. Most of the analyses to date are based on the Gauss-Seidal iterative method. Hence, there is need for further research in this direction which may result in efficient computer-codes.

\section{Conclusions}

For a pure metal, the variable domain methods, especially the ones based on boundary immobilization, will yield more accurate results than those based on fixed domain methods. On the other hand, the fixed domain method is very much easier to program because tracking of the interface is not needed. Thus, fixed domain methods, unlike variable domain methods, can be easily extended to multidimensional problems. For alloy solidification problems, fixed domain methods are always superior to variable domain methods. This is because of the existence of four different zones, namely, solid, eutectic, mush and liquid, in alloy solidification problems which can be easily incorporated through proper enthalpy-temperature relationships. While analysing melting and solidification problems with convection, the boundary immobilization method is the most accurate because of the precise description of the liquid region. All the techniques based on the fixed domain method are approximate. A porous media model with fixed domain formulation seems to be the most generalised one because of its suitability for alloy solidification problems.

Among the methods of solution, the explicit difference scheme is very simple and convenient. Because of numerical instability, the explicit difference scheme is useful only for melting and solidification problems with large Stefan numbers, e.g., the total transient is small. For other problems, implicit methods must be resorted to and systems of nonlinear algebraic equations will need to be solved.

On the basis of experience gained so far, numerical methods based on a timeimplicit solution of the fixed domain equation can be highly recommended for multidimensional melting and solidification problems.

The following topics involve unsolved problems of technological interest and therefore research on them would be very worthwhile. 
(i) Physical models to study heat and solute flow in the mushy region during alloy solidification.

(ii) Physical models to predict the geometry of the mushy region and subsequently derive the proper Darcy equation.

(iii) Mathematical models to account for convection effects during melting and solidification in a finite domain.

(iv) Solution procedures to enhance the rate of convergence.

(v) Improvement of fixed domain formulations to eliminate the stepwise increase of the dependent variable, e.g. to calculate proper heat fluxes at the phase change control volume.

We wish to record our sincere thanks to Prof. E C Subbarao, TRDDC, Pune for several fruitful discussions. We are grateful to Ms P Lobo for assistance. This work was supported by a project from the Defence Research Development Organisation, New Delhi.

\section{List of symbols}

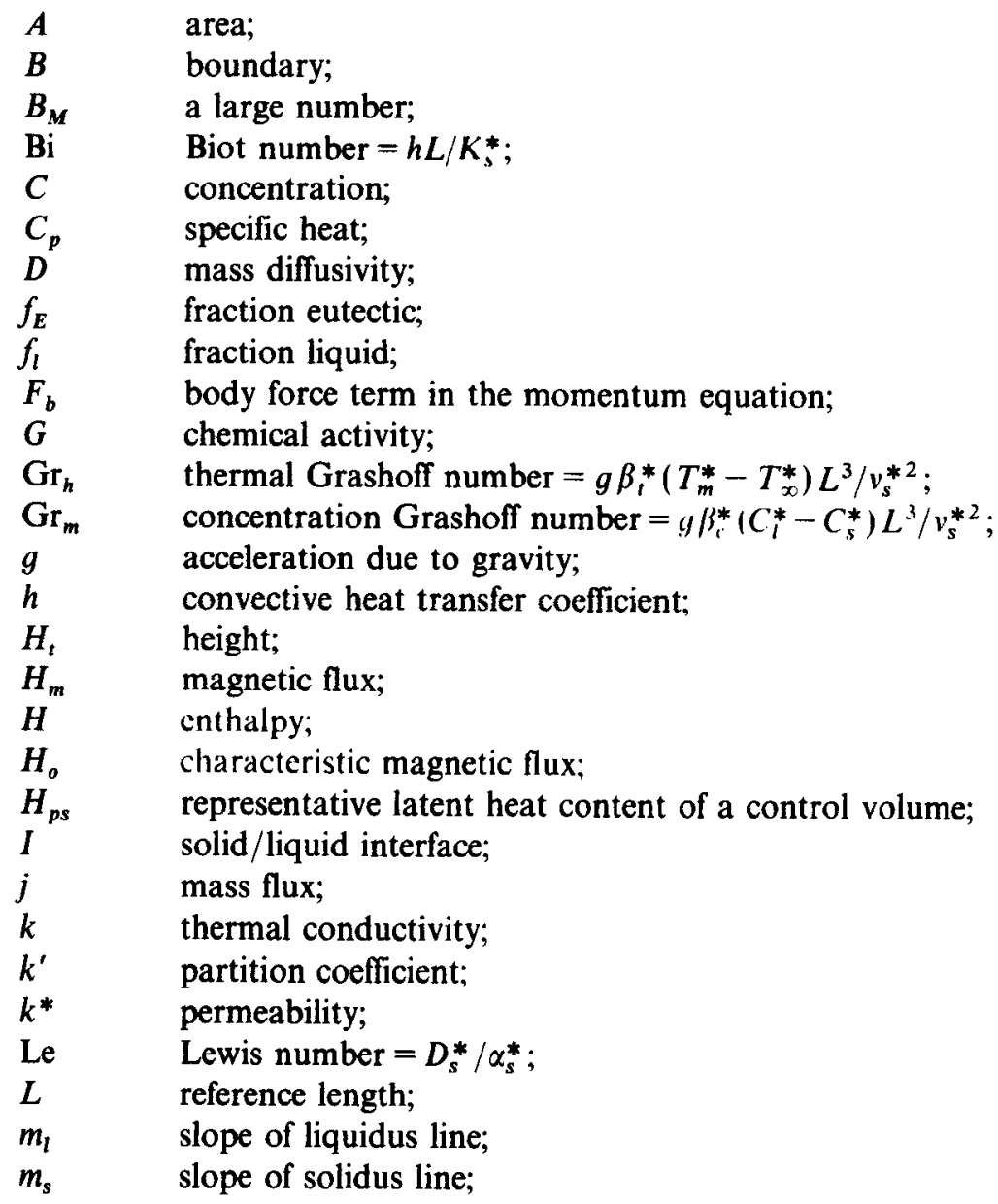


$M_{m} \quad$ magnetic Mach number $=U_{R} /\left(\mu_{m}^{*} H_{o} / \rho_{s}^{*}\right)^{1 / 2} ;$

$n$ normal direction;

$\mathrm{Nu} \quad$ Nusselt number $=h L / k_{l}^{*}$;

$p \quad$ pressure;

$\mathrm{Pe} \quad$ Peclet number;

$\operatorname{Pr} \quad$ Prandtl number $=v_{s}^{*} / \alpha_{s}^{*}$;

$q \quad$ heat flux;

$q_{b} \quad$ boundary heat flux;

$Q \quad$ total heat generation;

$r \quad$ space variable;

Re Reynolds number $=U_{R} L / v_{s}^{*}$;

$\mathrm{Ra} \quad$ Rayleigh number $=\beta_{t}^{*} g\left(T_{m}^{*}-T_{\infty}^{*}\right) L^{3} /\left(v_{s}^{*} \alpha_{s}^{*}\right)$;

$R_{F} \quad$ radiation factor $=\sigma \varepsilon_{R} L\left(T_{b}^{* 2}+T_{\infty}^{* 2}\right)\left(T_{b}^{*}+T_{\infty}^{*}\right) / k_{s}^{*}$;

$R_{S F} \quad$ surface tension factor $=\left(\partial \sigma_{s} / \partial T^{*}\right)\left[\left(T_{m}^{*}-T_{\infty}^{*}\right) /\left(\mu_{l}^{*} U_{R}\right)\right]$;

$R_{f r} \quad$ freezing rate;

$R \quad$ radius of curvature;

$S \quad$ entropy of melting;

$S \quad$ source term;

Sc $\quad$ Schmidt number $=v_{s}^{*} / D_{s}^{*}$;

Ste $\quad$ Stefan number $=C_{p}^{*}\left(T_{m}^{*}-T_{\infty}^{*}\right) / \lambda$;

$t \quad$ time;

$t_{f} \quad$ local solidification time;

$T \quad$ temperature;

$T_{b l} \quad$ bulk mean temperature of liquid;

$u, v, w \quad$ velocity;

$U_{R} \quad$ characteristic velocity;

$V \quad$ volume;

$X_{b} \quad$ Brody-Fleming's back diffusion parameter $=4 D_{s}^{*} t_{f} / L^{2}$;

$X_{c} \quad$ modified back diffusion parameter $=X_{b}\left[1-\exp \left(-1 / X_{b}\right)\right]-\frac{1}{2} \exp \left(-1 / 2 X_{b}\right)$;

$x, y, z \quad$ space variables;

$\alpha \quad$ thermal diffusivity;

$\beta_{c} \quad$ coefficient of thermal gradient due to concentration gradient;

$\beta_{t} \quad$ coefficient of volumetric expansion due to thermal gradient;

$\gamma \quad$ convergence factor;

$\Gamma \quad$ any transport property;

$\varepsilon \quad$ diffuse range;

$\varepsilon_{R} \quad$ emissivity;

$\eta, \xi \quad$ transformed space variables;

$\theta \quad$ nondimensional temperature;

$\lambda \quad$ latent heat of fusion; transformed space variable;

$\mu \quad$ dynamic viscosity;

$\mu_{m} \quad$ magnetic permeability;

$v \quad$ kinematic viscosity;

$\rho \quad$ density;

$\sigma \quad$ Stefan-Boltzmann constant;

$\sigma_{s} \quad$ surface tension;

$\tau \quad$ nondimensional time;

$\phi \quad$ any dependant variable;

$\psi \quad$ nondimensional enthalpy. 


$\begin{array}{ll}\text { Subscripts } & \\ \text { amb } & \text { ambient temperature; } \\ c & \text { mass transfer; } \\ e & \text { energy; } \\ \text { eff } & \text { effective value; } \\ E & \text { eutectic; } \\ i & \text { any grid point; } \\ I & \text { interface; } \\ k & \text { iteration level; } \\ l & \text { liquid; liquidus; } \\ m & \text { momentum; melting point; } \\ \text { mush } & \text { mushy region; } \\ R & \text { reference; } \\ s, S & \text { solid; } \\ \text { sat } & \text { saturated; } \\ \infty & \text { surrounding. }\end{array}$

\section{Superscripts}

$\begin{array}{ll}* & \text { dimensional value; } \\ k & \text { iteration level; } \\ n & \text { new time level; } \\ s & \text { saturated state. }\end{array}$

\section{References}

Albasiny E L 1956 Proc. IEEE B103: 158-162

Atthey D R 1974 J. Inst. Math. Its Appl. 13: 335-366

Bannerot R, Jamet P 1975 J. Comput. Phys. 18: 21-45

Bannerot R, Jamet P 1977 J. Comput. Phys. 25: 163-181

Basu B, Date A W 1987 On local vs. global implicitness during melting and solidification problems. presented at the 9th National Heat Mass Transfer Conference, Bangalore

Basu B. Sekhar J A 1988 Metall. Trans. (in press)

Basu B, Srinivasan J 1988 Int. J. Heat Mass Trans. (in press)

Baxter D C 1962 J. Heat Transfer C84: 317-326

Beckermann C. Viskanta R 1988 Physico-Chemical hydrodynamics 10: 195-213

Bell G E 1982 Int. J. Heat Mass Transfer 25: 587-589

Benard C, Gobin D, Martinez F 1985 J. Heat Transfer C107: 794-803

Benard C, Gobin D, Zanoli A 1986 Int. J. Heat Mass Transfer 29: 1669-1681

Brody H D, Flemings M C 1966 Trans. Metall. Soc. AIME 236: 615

Bonacina C. Comini G, Fasand A, Primicenid M 1975 Int. J. Heat Mass Transfer 16: 1825-1832

Chan C. Mazumder J, Chen M M 1983 Lasers in materials processing (ed.) E A Metzbower (Metals Park, Ohio: ASM)

Chan C, Mazumder J. Chen M M 1984 Metall. Trans. A15: 2175-2184

Chan C, Mazumder J, Chen M M 1985 Three-dimensional model for convection in laser melted pool. Presented at the International Congress on Lasers and Electro-optics, November, San Fransisco, CA

Chande T, Mazumder J 1985 J. Appl. Phys. 57: 2226-2232

Chiesa F M, Guthrie R I L 1974 J. Heat Transfer C96: 377-384

Clyne T W, Kurz W 1981 Metall. Trans. A12: 965

Comini G. Guidice S D. Lewis R W. Zienkiewiez O C 1974 Int. J. Numer. Methods Eng. 8: 613624

Crank J 1981 Numerical methods in heat transfer (eds) R W Lewis, K Morgan, O C Zienkiewiez (New York: John Wiley \& Sons)

Crank J. Crowley A B 1978 Int. J. Heat Mass Transfer 21: 393-398 
Crank J, Crowley A B 1979 Int. J. Heat Mass Transfer 22: 1331-1337

Crank J, Gupta R S 1975 Int. J. Heat Mass Transfer 18: 1101-1107

Crowley A B 1978 Int. J. Heat Mass Transfer 21: 215-219

Crowley A B, Ockendon J R 1979 Int. J. Heat Mass Transfer 22: 941-947

Douglas J, Gallie T M 1970 Duke Math. J. 22: 557-571

Duda J L. Malone M F, Notten R H, Ventas J S 1975 Int. J. Heat Mass Transfer 18: 901-910

Dusinberre G M 1945 Trans. ASME 67: 703-710

Eyres N R, Hartree D R, Ingham J, Jackson R, Sarjant R J, Wagstaff J B 1946 Philos. Trans. R. Soc. 240: $1-57$

Fix G J 1978 Moving boundary problems (eds) D G Walson, A D Solomon, P T Boggs (New York: Academic Press)

Flemings M C 1974 Solidification processing (New York: McGraw-Hill)

Gadgil A, Gobin D 1984 J. Heat Transfer C106: 21-26

Gartling D K 1980 Computer methods in fluids (ed.) K Mörgan (London: Pentech)

Godbole D B, Date A W 1985 Numerical algorithm for solving systems resulting from elliptic equations of heat transfer, Proc. 8th National Heat Mass Transfer Conf., Paper No: HMT-D19-85, pp. 325-329

Golder D, Guy A G 1975 Moving boundary problems in heat flow and diffusion (New York: Clarendon)

Goodling J S, Khader M S 1974 J. Heat Transfer C96: 114-115

Goodman T R 1958 Trans. ASME 80: 335-358

Goodrich L E 1978 Int. J. Heat Mass Transfer 21: 615-621

Grange B W, Viskanta R, Stevenson W H 1976 Int. J. Heat Mass Transfer 19: 373-384

Gupta R S, Kumar A 1985 Int. J. Heat Mass Transfer 28: 1355-1366

Gupta R S, Kumar D 1981 Int. J. Heat Mass Transfer 24: 251-259

Heitz W L, Westwater J W 1970 Int. J. Heat Mass Transfer 13: 1371-1375

Hsu S C, Chakraborty S, Mehrabian R 1978 Metall. Trans. B9: 221-229

Hsu S C, Kou S, Mehrabian R 1980 Metall. Trans. B11: 24-28

Hsu C F, Sparrow E M, Patankar S V 1981 Int. J. Heat Mass Transfer 24: 1335-1343

Huber A 1939 Z. Angew. Math. Mech. 19: 1-20

Hunt J D, McCartney D G 1987 Acta Metall. 35: 89

Huppert H E, Worster M G 1985 Nature (London) 314: 703-707

Jamet P 1978 SIAM J. Numer. Anal. 15: 912-928

Kroeger P G, Ostrach S 1974 Int. J. Heat Mass Transfer 17: 1191-1207

Koh J C Y, Price J F, Coloney R 1969 Prog. Heat Mass Transfer 2: 225-247

Kou S, Hsu S C, Mehrabian R 1981 Metall. Trans. B12: 33-45

Kou S, Sun D K 1985 Metall. Trans. A16: 203-213

Kou S, Wang Y H 1986a Metall. Trans. A17: 2265-2270

Kou S, Wang Y H 1986b Metall. Trans. A17: 2271-2277

Lazardis A 1970 Int. J. Heat Mass Transfer 13: 1459-1477

Mehrabian R, Keane M, Flemmings M C 1970 Metall. Trans. 1: 1209-1220

Meyer G H 1971 SIAM J. Numer. Anal. 8: 80 96

Mcyer G H 1978 Moving boundary problems (eds) D G Wilson, A D Solomon, P T Boggs (New York: Academic Press)

Meyer G H 1981 Int. J. Heat Mass Transfer 24: 778-781

Morgan K 1981 Comput. Methods Appl. Eng. 28: 275-284

Morgan M, Lewis R W, Zienkiewiez O C 1978 Int. J. Numer. Methods Eng. 12: 1191-1195

Murray W D, Landis F $1954 \mathrm{~J}$. Heat Transfer C81: 105-112

Oreper G M, Szekely J 1984 J. Fluid Mech. 147: 53-79

Oreper G M, Szekely J, Eager T W 1986 Metall Trans. B17: 735-744

Patankar S V 1980 Numerical heat transfer and fluid flow (New York: McGraw-Hill)

Patel P D 1968 AIAA J. 6: 2454

Poots G 1962 Int. J. Heat Mass Transfer 5: 339-348

Price P H. Slack M R 1954 Br. J. Appl. Phys. 5: 285-287

Ramachandran N. Gupta J P. Jaluria Y 1981 Numer. Hoat Transfer 4: 469-484

Ramachandran N, Gupta J P, Jaluria Y 1982 Int. J. Heat Mass Transfer 24: 187-194

Ramarao K V, Sekhar J A 1987 Acta Metall. 35: 81-87

Rao P R, Sastri V M K 1983 Numer. Heat Transfer 6: 103-114

Rao P R. Sastri V M K 1984 Numer. Heat Transfer 7: 2077-2084 
Rathjen K A, Jiji L M 1971 J. Heat Transfer C93: 101-114

Reddy G S, Sekhar J 1985 J. Mater. Sci. 20: 3535-3544

Rieger H, Projohn V, Beer M 1982 Int. J. Heat Mass Transfer 25: 137-147

Roach P J 1976 Computational fluid dynamics (Albuquerque: Hermosa)

Rolphe III W D, Bathe K J 1982 Int. J. Numer. Methods Eng. 18: 119-134

Saitoh T 1978 J. Heat Transfer C100: 294-299

Sekhar J A, Kou S, Mehrabian R 1983 Metall. Trans. A14: 1169-1177

Shamsundar N 1978 Moving boundary problems (eds) D G Wilson, A D Solomon, P T Boggs (New York: Academic Press)

Shamsundar N, Sparrow E M 1975a J. Heat Transfer C97: 350-357

Shamsundar N, Sparrow E M 1975b J. Heat Transfer C97: 550-557

Spaid F W, Charwat A F. Redekopp L G, Rasen R 1971 Int. J. Heat Mass Transfer 14: 673 687

Sparrow E M, Hsu C F 1981 Int. J. Heat Mass Transfer 24: 1344-1357

Sparrow E M, Patankar S V, Ramdhyani S 1977 J. Heat Transfer C99: 520-526

Sparrow E M, Souza Mendis P 1982 Int. J. Heat Mass Transfer 25: 293-297

Springer G S, Olson D R 1962 Trans. ASME Paper No 62-WA-246

Sproster J L 1981 Int. J. Heat Mass Transfer 24: 1493-1501

Stefan J 1891 Ann. Phys. Chem. 42: 269-286

Szekely J, Chabbra P 1970 Metall. Trans. 1: 1195-1203

Szekely J, Themlis N J 1970 Rate phenomena in process metallurgy (New York: Wiley Interscience) chap. 10

Tien R H, Geiger G E 1967 J. Heat Transfer C90: 27-31

Tien L C, Wilkes J O 1970 Proc. IV Int. Heat Trans. Conference, Paris (eds) U Grigull, E Hahne (Amsterdam: Elsevier) Paper No. Cu 2.12

Voller V R 1985 IMA J. Numer. Anal. 5: 201-214

Voller V R 1987 Appl. Math. Modelling 11: 110-116

Voller V R, Cross M 1981a Int. J. Heat Mass Transfer 24: 545-556

Voller V R, Cross M 1981b Int. J. Heat Mass Transfer 24: 1457-1461

Voller V R, Cross M, Marktos N C 1987 Int. J. Numer. Methods Eng. 24: 271-284

Voller V R, Cross M, Walton P 1979 Numerical methods in thermal problems (eds) R W Lewis and K Morgan (London: Pineridge)

Worster M G 1986 J. Fluid Mech. 167: 481-501 